\title{
Method for computing waveguide scattering matrices in vicinity of thresholds
}

\author{
B. A. Plamenevskii, A. S. Poretskii, and O.V. Sarafanov *
}

April 11, 2014

\begin{abstract}
A waveguide occupies a domain $G$ in $\mathbb{R}^{n+1}, n \geq 1$, having several cylindrical outlets to infinity. The waveguide is described by the Dirichlet problem for the Helmholtz equation. The scattering matrix $S(\mu)$ with spectral parameter $\mu$ changes its size wnen $\mu$ crosses a threshold. To calculate $S(\mu)$ in a neighborhood of a threshold, we introduce an "augmented" scattering matrix $\mathcal{S}(\mu)$ that keeps its size near the threshold, where the matrix $\mathcal{S}(\mu)$ is analytic in $\mu$. A minimizer of a quadratic functional $J^{R}(\cdot, \mu)$ serves as an approximation to a row of the matrix $\mathcal{S}(\mu)$. To construct such a functional, we solve an auxiliary boundary value problem in the bounded domain obtained by cutting off, at a distance $R$, the waveguide outlets to infinity. As $R \rightarrow \infty$, the minimizer $a(R, \mu)$ at exponential rate tends to the corresponding row of $\mathcal{S}(\mu)$ uniformly with respect to $\mu$ in a neighborhood of the threshold. The neighborhood may contain some waveguide eigenvalues corresponding to eigenfunctions exponentially decaying at infinity. Finally, we express the elements of the "ordinary" scattering matrix $S(\mu)$ through those of the augmented matrix $\mathcal{S}(\mu)$.

If an interval $\left[\mu_{1}, \mu_{2}\right]$ of the continuous spectrum contains no thresholds, the corresponding functional $J^{R}(\cdot, \mu)$ should be defined for the ordinary matrix $S(\mu)$ and, as $R \rightarrow \infty$, its minimizer $a(R, \mu)$ tends to the row of the scattering matrix at exponential rate uniformly with respect to $\mu \in\left[\mu_{1}, \mu_{2}\right]$.
\end{abstract}

\section{Introduction}

The waveguide considered in the paper occupies a domain $G$ in the space $\mathbb{R}^{n+1}$ with smooth boundary $\partial G$ and finitely many cylindrical outlets to infinity (cylindrical ends). By this we mean that, outside a large ball centered at the origin, the domain $G$ coincides with the union of nonoverlapping semicylinders $\Pi_{+}^{1}, \ldots, \Pi_{+}^{\mathcal{T}}$; here $\Pi_{+}^{p}=\left\{\left(y^{p}, t^{p}\right): y^{p} \in \Omega^{p}, t^{p}>0\right\}$, the $\left(y^{p}, t^{p}\right)$ are local coordinates in $\Pi_{+}^{p}$, and the cross-section $\Omega^{p}$ of the cylinder $\Pi^{p}$ is a bounded domain in $\mathbb{R}^{n}$. The waveguide is described by the Dirichlet problem for the operator $-\Delta-\mu$, where $\mu$ is a spectral parameter and $\Delta$ is the Laplace operator. The continuous spectrum of the problem coincides with semiaxis $\left\{\mu \in \mathbb{R}: \tau_{1} \leq \mu\right\}$, the $\tau_{1}$ being a positive number.

*The authors were supported by Grants RFBR-12-01-00247a and Scientific Schools-357.2012.1 
For every point $\mu \in\left[\tau_{1},+\infty\right)$ there exist $\varkappa(\mu)$ solutions, $\varkappa(\mu)<\infty$, to the homogeneous problem

$$
\begin{array}{r}
-\Delta u(x)-\mu u(x)=0, \quad x \in G, \\
u(x)=0, \quad x \in \partial G,
\end{array}
$$

satisfying $|u(x)| \leq \operatorname{Const}(1+|x|)$ in $G$ and linearly independent modulo $L_{2}(G)$. Such solutions are called the continuous spectrum eigenfunctions and the number $\varkappa(\mu)$ is called the multiplicity of the continuous spectrum. The threshold values (thresholds) form a sequence $\tau_{1}<\tau_{2}, \ldots, \tau_{n} \rightarrow+\infty$. The multiplicity $\varkappa(\mu)$ is constant on every interval $\left[\mu^{\prime}, \mu^{\prime \prime}\right]$ of the continuous spectrum containing no threshold. The function $\mu \mapsto \varkappa(\mu)$ has discontinuity at every threshold being continuous from the right. This is an increasing function, so $\varkappa(\mu) \rightarrow$ $+\infty$ as $\mu \rightarrow+\infty$.

There may also exist eigenvalues of problem (1.1) embedded into the continuous spectrum whose eigenfunctions belong to $L_{2}(G)$. Such an eigenfunction exponentially decays at infinity, any eigenvalue is of finite multiplicity, and the eigenvalues can only accumulate at infinity. An eigenvalue $\mu_{0}$ does not affect the multiplicity of the continuous spectrum at $\mu_{0}$ because $\varkappa\left(\mu_{0}\right)$ takes into account only linear independence modulo $L_{2}(G)$. If $\mu$ is not an eigenvalue, then the linear independence modulo $L_{2}(G)$ in the definition of $\varkappa(\mu)$ can be changed for the usual linear independence.

It is known [1] that, for every $\mu \in\left[\tau_{1},+\infty\right]$ in the space of continuous spectrum eigenfunctions, there exists a basis $Y_{1}(\cdot, \mu), \ldots, Y_{M}(\cdot, \mu)$ modulo $L_{2}(G)$ such that

$$
Y_{j}(x, \mu)=u_{j}^{+}(x, \mu)+\sum_{k=1}^{\varkappa(\mu)} S_{j k}(\mu) u_{k}^{-}(x, \mu)+O\left(e^{-\varepsilon|x|}\right)
$$

for $|x| \rightarrow \infty$ and $j=1, \ldots, \varkappa(\mu)$; here $\varepsilon$ is a sufficiently small number, $u_{j}^{+}(\cdot, \mu)$ is an "incoming" wave, and $u_{j}^{-}(\cdot, \mu)$ is an "outgoing" one (precise definitions see in Section 2.2). The matrix $S(\mu)=\left\|S_{j k}(\mu)\right\|$ is unitary; it is called the scattering matrix.

In [2] and [3], a method for approximate computing the matrix $S(\mu)$ was discussed under the condition that $\mu$ varies on an interval $\left[\mu^{\prime}, \mu^{\prime \prime}\right]$ of the continuous spectrum containing no thresholds. Briefly, a minimizer $a(R, \mu)$ of a quadratic functional $J_{l}^{R}(\cdot, \mu)$ was chosen as an approximation to the $l$-th row $S_{l}(\mu)=\left(S_{l, 1}(\mu), \ldots, S_{l, M}(\mu)\right)$ of the scattering matrix $S(\mu)$. To construct such a functional, one has to solve an auxiliary boundary value problem in the bounded domain $G^{R}$ obtained from $G$ by cutting off the cylindrical ends at a sufficiently large distance $R$ from the coordinate origin. For $R \geqslant R_{0}$ and all $\mu \in\left[\mu^{\prime}, \mu^{\prime \prime}\right]$ the estimate

$$
\left\|a(R, \mu)-S_{l}(\mu)\right\| \leqslant C e^{-\gamma R}
$$

was proved with positive number $\gamma$ and a constant $C$ independent of $R$ and $\mu$. In [2], the twodimensional waveguides and the Helmholtz operator were considered, while the waveguides of arbitrary dimensions and the self-adjoint elliptic systems of any order were discussed in [3]. The approach suggested in [3] turns out to be new for the Helmholtz operator as well and more simple than that in [2].

The present paper is devoted to a method for approximate computing the scattering matrices in a neighborhood of the threshods. Let us outline the method. We assume $\tau^{\prime}<\tau^{\prime \prime}$ 
to be thresholds of problem (1.1) such that the interval $\left(\tau^{\prime}, \tau^{\prime \prime}\right)$ contains the only threshold $\tau$. We also suppose that the three thresholds relate to the same cylindrical end. We intend to (approximately) calculate the scattering matrix $S(\mu)$ in (1.2) for $\mu \in\left[\mu^{\prime}, \mu^{\prime \prime}\right]$ with $\tau \in$ $\left[\mu^{\prime}, \mu^{\prime \prime}\right] \subset\left(\tau^{\prime}, \tau^{\prime \prime}\right)$.

On the interval $\left(\tau, \tau^{\prime \prime}\right)$, one can choose a basis of incoming $w_{1}^{+}(\cdot, \mu), \ldots, w_{\varkappa}^{+}(\cdot, \mu)$ and outgoing $w_{1}^{-}(\cdot, \mu), \ldots, w_{\varkappa}^{-}(\cdot, \mu)$ waves with analytic functions $\left(\tau, \tau^{\prime \prime}\right) \ni \mu \mapsto w_{j}^{ \pm}(\cdot, \mu)$, which admit the analytic continuation to $\left(\tau^{\prime}, \tau^{\prime \prime}\right)$; here $\varkappa=\varkappa\left(\mu^{\prime \prime}\right)$ (recall that $\varkappa(\mu)=$ const for $\left.\mu \in\left[\tau, \tau^{\prime \prime}\right)\right)$. Such a basis is called stable at the threshold $\tau$. For $\mu \in\left(\tau^{\prime}, \tau\right)$, some incoming waves and the same number of outgoing waves turn out to be exponentially growing as $x \rightarrow \infty$. On the interval $\left(\tau, \tau^{\prime \prime}\right)$, in the space of continuous spectrum eigenfunctions there exists a basis $\mathcal{Y}_{1}(\cdot, \mu), \ldots, \mathcal{Y}_{\varkappa}(\cdot, \mu)$ satisfying the conditions

$$
\mathcal{Y}_{j}(x, \mu)=w_{j}^{+}(x, \mu)-\sum_{k=1}^{M} \mathcal{S}_{j k}(\mu) w_{k}^{-}(x, \mu)+O\left(e^{-\varepsilon|x|}\right) .
$$

The functions $\mu \mapsto \mathcal{Y}_{j}(\cdot, \mu)$ and $\mu \mapsto \mathcal{S}_{j k}(\mu)$ are analytic and admit the analytic continuation to $\left(\tau^{\prime}, \tau^{\prime \prime}\right)$. In contrust to $S(\mu)$, the new matrix $\mathcal{S}(\mu)=\left\|\mathcal{S}_{j k}(\mu)\right\|$ keeps its size on the interval; the matrix is unitary for all $\mu \in\left(\tau^{\prime}, \tau^{\prime \prime}\right)$. The entries of $S(\mu)$ can be expressed in terms only related to the matrix $\mathcal{S}(\mu)$. In particular this enables us to prove the existence of finite limits $\lim S(\mu)$ as $\mu \rightarrow \tau \pm 0$, to calculate the limits, and in essence to reduce the approximate calculation of the matrix $S(\mu)$ with $\mu \in\left[\mu^{\prime}, \mu^{\prime \prime}\right]$ to that of the augmented matrix $\mathcal{S}(\mu)$. As an approximation to a row of $\mathcal{S}(\mu)$, we take a minimizer of a quadratic functional $\mathcal{J}^{R}(\cdot, \mu)$. To construct such a functional we use a boundary value problem in the bounded domain $G^{R}$ obtained from $G$ by cutting off the cylindrical ends at a distance $R$. We set

$$
\begin{aligned}
& \Pi_{+}^{r, R}=\left\{\left(y^{r}, t^{r}\right) \in \Pi^{r}: t^{r}>R\right\}, \quad G^{R}=G \backslash \cup_{r=1}^{\mathcal{T}} \Pi_{+}^{r, R}, \\
& \partial G^{R} \backslash \partial G=\Gamma^{R}=\cup_{r} \Gamma^{r, R}, \quad \Gamma^{r, R}=\left\{\left(y^{r}, t^{r}\right) \in \Pi^{r}: t^{r}=R\right\}
\end{aligned}
$$

for large $R$ and introduce the boundary value problem

$$
\begin{aligned}
-\Delta \mathcal{X}_{j}^{R}-\mu \mathcal{X}_{j}^{R} & =0, \quad x \in G^{R} \\
\mathcal{X}_{j}^{R} & =0 \quad x \in \partial G^{R} \backslash \Gamma^{R} \\
\left(-\partial_{n}+i \zeta\right) \mathcal{X}_{j}^{R} & =\left(-\partial_{n}+i \zeta\right)\left(w_{j}^{+}+\sum_{k=1}^{M} a_{k} w_{k}^{-}\right), x \in \Gamma^{R},
\end{aligned}
$$

where $w_{j}^{ \pm}$is a stable basis in the space of waves, $\zeta \in \mathbb{R} \backslash\{0\}$ is an arbitrary fixed number, and $a_{k}$ are complex numbers. As approximation to the row $\left(\mathcal{S}_{j 1}(\mu), \ldots, \mathcal{S}_{j M}(\mu)\right.$, we take the minimizer $a^{0}(R, \mu)=\left(a_{1}^{0}(R, \mu), \ldots, a_{M}^{0}(R, \mu)\right)$ of the functional

$$
\mathcal{J}_{j}^{R}\left(a_{1}, \ldots, a_{M}\right)=\left\|\mathcal{X}_{j}^{R}(\cdot, \mu)-w_{j}^{+}(\cdot, \mu)-\sum_{k=1}^{M} a_{k} w_{k}^{-}(\cdot, \mu) ; L_{2}\left(\Gamma^{R}\right)\right\|^{2},
$$

where $\mathcal{X}_{j}^{R}(\cdot, \mu)$ is a solution to the boundary value problem. If $\tau \in\left[\mu^{\prime}, \mu^{\prime \prime}\right] \subset\left(\tau^{\prime}, \tau^{\prime \prime}\right)$, then the inequality

$$
\left\|a(R, \mu)-\mathcal{S}_{j}(\mu)\right\| \leqslant C(\Lambda) e^{-\Lambda R}
$$


holds for all $\mu \in\left[\mu^{\prime}, \mu^{\prime \prime}\right]$ and $R \geqslant R_{0}$ with positive constants $\Lambda$ and $C(\Lambda)$ independent of $\mu$ and $R$.

Note that the use of stable bases is not uncommon in asymptotic studies of various "threshold" situations. In this connection we refer to [4] и [5], where the asymptotics of solutions to elliptic boundary value problems was investigated near singularities of the boundary (see also References in the papers). In [6], the asymptotics of the scattering matrix for a two dimensional diffraction grating was justified, in essence, with the help of a stable basis in the space of waves.

In the present paper, $\S 2$ is devoted to constructing a stable basis of waves in a neighborhood of a threshold for the waveguide in the domain $G$. The continuous spectrum eigenfunctions and the scattering matrices $S(\mu)$ and $\mathcal{S}(\mu)$ are introduced in в $\S 3$; here we also prove the analyticity of the matrices on the corresponding intervals of the continuous spectrum. We describe the connections between the matrices $S(\mu)$ and $\mathcal{S}(\mu)$ and calculate the one-sided limits of $S(\mu)$ at a threshold in $\S 4$. The last $\S 5$ contains the statement and justification of the method for approximate computation of the scattering matrices.

\section{Augmented space of waves}

\subsection{The waves in a cylinder}

In the cylinder $\Pi=\left\{(y, t): y=\left(y_{1}, \ldots, y_{n}\right) \in \Omega, t \in \mathbb{R}\right\}$ we consider the problem

$$
\begin{aligned}
(-\Delta-\mu) u(y, t) & =0, \quad(y, t) \in \Pi, \\
u(y, t) & =0 \quad(y, t) \in \partial \Pi,
\end{aligned}
$$

where

$$
\Delta=\Delta_{y}+\partial_{t}^{2}, \quad \Delta_{y}=\sum_{j=1}^{n} \partial_{j}^{2}, \quad \partial_{j}=\partial / \partial y_{j}
$$

Let us connect with problem (2.1) an operator pencil $\mathbb{C} \ni \lambda \mapsto \mathfrak{A}(\lambda, \mu)$ setting

$$
\mathfrak{A}(\lambda, \mu) v(y)=\left(-\Delta_{y}+\lambda^{2}-\mu\right) v(y), \quad y \in \Omega ;\left.\quad v\right|_{\partial \Omega}=0 .
$$

We also consider the problem

$$
\begin{aligned}
\left(-\Delta_{y}-\mu\right) v(y) & =0, \quad y \in \Omega, \\
v(y) & =0, \quad y \in \partial \Omega .
\end{aligned}
$$

The eigenvalues of problem (2.3) are called the thresholds of problem (2.1). The thresholds form a positive sequence $\tau_{1}<\tau_{2}<\ldots$, which strictly increases to infinity. Let us introduce the nondecreasing sequence $\left\{\mu_{k}\right\}_{k=1}^{\infty}$ of the eigenvalues of problem (2.3), counted according to their multiplicity (generally, the numbering of $\tau_{l}$ and that of $\mu_{k}$ are different; every $\mu_{k}$ coincides with one of the thresholds $\tau_{l}$ ). We assume that the corresponding eigenvectors $\varphi_{k}$ are orthogonal and normalized by the condition

$$
\int_{\Omega} \varphi_{k}(y) \overline{\varphi_{k}(y)} d y=1
$$


The spectrum of the operator pencil (for every fixed $\mu \in \mathbb{R}$ ) consists of isolated eigenvalues on the axes of complex plane.For any $\mu$ the eigenvalues $\lambda_{k}^{ \pm}$of the pencil $\lambda \mapsto \mathfrak{A}(\lambda, \mu)$ are defined by $\lambda_{k}^{ \pm}= \pm\left(\mu-\mu_{k}\right)^{1 / 2}$. If $\lambda_{k}^{ \pm} \neq 0$, to the eigenvalues $\lambda_{k}^{ \pm}$there corresponds the same eigenvector $\varphi_{k}$, which is also an eigenvector of problem (2.3) corresponding to the eigenvalue $\mu_{k}$. There is no generalized eigenvector in the case. If $\mu_{k-1}<\mu<\mu_{k}$, then $\lambda_{k}^{ \pm}, \lambda_{k+1}^{ \pm}, \ldots$ are imaginary and $\lambda_{1}^{ \pm}, \ldots, \lambda_{k-1}^{ \pm}$are real. In the case $\mu=\mu_{k}$, to the eigenvalue $0=\lambda_{k}^{+}=\lambda_{k}^{-}$ there corresponds a Jordan chain $\varphi^{0}, \varphi_{k}^{1}$, where $\varphi_{k}^{0}$ is an eigenvector and $\varphi_{k}^{1}$ is a generalized eigenvector.

We fix a real $\mu \neq \mu_{k}, k=1,2, \ldots$, that is, the $\mu$ is not a threshold, and introduce the linear complex space spanned by the functions

$$
(y, t) \mapsto \exp \left(i \lambda_{k}^{ \pm} t\right) \varphi_{k}(y)
$$

with real $\lambda_{k}^{ \pm}= \pm\left(\mu-\mu_{k}\right)^{1 / 2}$; the functions (2.5) satisfy (2.1). We denote the space by $W(\mu)$ and will call it the space of waves. Its dimension is equal to the doubled number of $\mu_{k}$ (counted according to their multiplicities) such that $\mu_{k}<\mu$. The functions

$$
u_{k}^{ \pm}(y, t ; \mu)=\left(2\left|\lambda_{k}^{\mp}\right|\right)^{-1 / 2} \exp \left(i \lambda_{k}^{\mp} t\right) \varphi_{k}(y)
$$

form a basis in $W(\mu)$. We call $u_{k}^{+}(\cdot, \mu)$ a wave incoming from $+\infty$, and $u_{k}^{-}(\cdot, \mu)$ a wave outgoing to $+\infty$.

Assume now that $\mu=\tau$ is a threshold and, consequently, $\mu$ is an eigenvalue of $(2.3)$ with multiplicity $\varkappa \geq 1$. Then $\varkappa$ numbers $\mu_{l}$ satisfy $\mu_{l}=\tau$. For each $l$ the functions $\exp \left(i \lambda_{l}^{+} t\right) \varphi_{l}(y)$ and $\exp \left(i \lambda_{l}^{-} t\right) \varphi_{l}(y)$ coincide. Therefore the number of linearly independent functions of the form (2.5) for $\mu=\tau$ is $\varkappa$ less than the number of such functions for $\mu$ satisfying $\tau<\mu<\tau+\beta$ with small $\beta>0$. However for a more general notion of the waves, the dimension of the space $W(\mu)$ is continuous from the right at the threshold. In such a case the definition of incoming and outgoing waves is based on energy reasons as in the Sommerfeld and Mandelstamm principles.

For the definition we introduce the form

$$
\begin{aligned}
q_{N}(u, v): & ((-\Delta-\mu) u, v)_{\Pi(N)}+\left(u,-\partial_{\nu} v\right)_{\partial \Pi(N) \cap \partial \Pi} \\
& -(u,(-\Delta-\mu) v)_{\Pi(N)}-\left(-\partial_{\nu} u, v\right)_{\partial \Pi(N) \cap \partial \Pi,}
\end{aligned}
$$

where $\Pi(N)=\{(y, t) \in \Pi: t<N\}$, the number $\mu \in \mathbb{R}$ is for the time being not a threshold, $u=\chi f$ and $v=\chi g$, while $f$ and $g$ are any of the functions (2.6)corresponding to real $\lambda_{k}^{ \pm}(\mu)$ (possibly with distinct indices); $\chi$ stands for a smooth cut-off function, $\chi(t)=0$ for $t<T-1$ and $\chi(t)=1$ for $t>T$ with $T<N$. Integrating by parts, we see that

$$
\begin{aligned}
& i q_{N}\left(\chi u_{k}^{ \pm}, \chi u_{l}^{\mp}\right)=0 \text { for all } k, l, \\
& i q_{N}\left(\chi u_{k}^{ \pm}, \chi u_{l}^{ \pm}\right)=\mp \delta_{k l},
\end{aligned}
$$

so the result is independent of $N$ and $\chi$; in what follows we drop $N$ but keep $\chi$. We name the wave $u_{k}^{+}\left(u_{k}^{-}\right)$incoming (outgoing) for $-(+)$on the right in (2.9) and obtain the definition of incoming (outgoing) waves equivalent to the old definition.

We are going to consruct a basis in the (augmented) space of waves "stable at a threshold". Let $\mu \in \mathbb{R}$ be a regular value of the spectral paremeter of problem (2.3) and 
$\mu_{m}$ the eigenvalue with the greatest number satisfying $\mu_{m}<\mu$. We also assume that $\mu_{l}<$ $\mu_{l+1}=\cdots=\mu_{m}$. Then the numbers $\tau^{\prime}:=\mu_{l}, \tau:=\mu_{l+1}=\cdots=\mu_{m}$, and $\tau^{\prime \prime}:=\mu_{m+1}$ turn out to be three successive thresholds $\tau^{\prime}<\tau<\tau^{\prime \prime}$ of problem (2.1) in the cylinder $\Pi$. (We discuss the general situation; the cases $l+1=m, m=1$, and so on, can be considered with evident simplifications.)

We set

$$
\begin{aligned}
w_{k}^{ \pm}(y, t ; \mu) & =2^{-1 / 2}\left(\frac{e^{i t \sqrt{\mu-\mu_{k}}}+e^{-i t \sqrt{\mu-\mu_{k}}}}{2} \mp \frac{e^{i t \sqrt{\mu-\mu_{k}}}-e^{-i t \sqrt{\mu-\mu_{k}}}}{2 \sqrt{\mu-\mu_{k}}}\right) \varphi_{k}(y), \\
w_{p}^{ \pm}(y, t ; \mu) & =u_{p}^{ \pm}(y, t ; \mu),
\end{aligned}
$$

where $k=l+1, \ldots, m, p=1, \ldots, l$, and $u_{p}^{ \pm}$are defined in (2.6).

Proposition 2.1. The functions $\mu \mapsto w_{k}^{ \pm}(y, t ; \mu), k=l+1, \ldots, m$, admit the analytic continuation to the whole complex plane.These analytic functions smoothly depend on the parameters $y \in \bar{\Omega}$ and $t \in \mathbb{R}$ (i.e., any derivatives in $y$ and $t$ are analytic functions as well).

The functions $\mu \mapsto w_{p}^{ \pm}(y, t ; \mu)$ are analytic on the complex plane with cut along the ray $\left\{\mu \in \mathbb{R}:-\infty<\mu \leq \mu_{p}\right\}, p=1, \ldots, l$; they smoothly depend on $y$ and $t$.

All the functions $w_{k}^{ \pm}, k=1, \ldots, m$, are solutions to problem (2.1). For every $\mu$ in $\left(\tau^{\prime}<\mu<+\infty\right)$ the functions (2.10), (2.11) satisfy the orthogonality and normalization conditions

$$
\begin{aligned}
& i q\left(\chi w_{r}^{ \pm}(\cdot ; \mu), \chi w_{s}^{\mp}(\cdot ; \mu)\right)=0 \quad \text { for all } r, s=1, \ldots, m, \\
& i q\left(\chi w_{r}^{ \pm}(\cdot ; \mu), \chi w_{s}^{ \pm}(\cdot ; \mu)\right)=\mp \delta_{r s} .
\end{aligned}
$$

Proof. The first and second fractions in the parentheses in (2.10) can be decomposed in the series

$$
\sum_{l \geq 0} \frac{\left(\mu_{k}-\mu\right)^{l} t^{2 l}}{(2 l) !} \text { and it } \sum_{l \geq 0} \frac{\left(\mu_{k}-\mu\right)^{l} t^{2 l}}{(2 l+1) !}
$$

which are absolutely and uniformly convergent on any compact $K \subset\{(\mu, t): \mu \in \mathbb{C}, t \in \mathbb{R}\}$. This implies the analyticity properties of $w_{k}^{ \pm}(y, t ; \mu)$ for $k=l+1, \ldots, m$. The corresponding assertions about $w_{p}^{ \pm}(y, t ; \mu)$ with $p=1, \ldots, l$ are evident.

It remains to verify the orthogonality and normalization conditions. We first assume that $\mu>\tau$ and consider, for instance, (2.13). If $r$ and $s$ are distinct then the equalities (2.13) follow from the orthogonality of $\varphi_{r}$ and $\varphi_{s}$ (as well as (2.8)and (2.9)). In the case $r=s \leq l,(2.9)$ contains the needed formula. Finally assume that $r=s>l$ and substitute the expressions (2.10) into $q\left(\chi w_{r}^{ \pm}, \chi w_{s}^{ \pm}\right)$. Setting $\lambda:=\sqrt{\mu-\tau}$, we obtain

$$
\begin{aligned}
i q\left(\chi w_{s}^{ \pm}, \chi w_{s}^{ \pm}\right) & =\lambda^{-2}\left((\lambda \pm 1)(\lambda \mp 1) i q^{+-}+(\lambda \mp 1)(\lambda \pm 1) i q^{-+}\right. \\
& \left.+(\lambda \mp 1)^{2} i q^{++}+(\lambda \pm 1)^{2} i q^{--}\right),
\end{aligned}
$$

where, for example, $q^{+-}=2^{-3} q\left(\chi e^{i t \lambda} \varphi_{s}, \chi e^{-i t \lambda} \varphi_{s}\right)$, and so on. Taking account of (2.6), (2.8), and (2.9), we arrive at (2.13).

We now consider the function

$$
\begin{aligned}
\mathbb{C} \ni \mu \mapsto q_{N}(u, v ; \mu): & ((-\Delta-\mu) u, v)_{\Pi(N)}+\left(u,-\partial_{\nu} v\right)_{\partial \Pi(N) \cap \partial \Pi} \\
& -(u,(-\Delta-\bar{\mu}) v)_{\Pi(N)}-\left(-\partial_{\nu} u, v\right)_{\partial \Pi(N) \cap \partial \Pi,}
\end{aligned}
$$


where $\Pi(N), N$, and $\chi$ are the same as in $(2.7), u=\chi w_{r}^{ \pm}(\cdot ; \mu)$, and $\left.v=\chi w_{s}^{\mp}(\cdot ; \bar{\mu})\right)$. Since $u$ and $\bar{v}$ are analytic, the function $\mu \mapsto q_{N}(u, v ; \mu)$ is analytic as well. Therefore, the equalities (2.13) (with $r=s>l$ ) are valid for all $\mu \in \mathbb{C}$.

From $(2.10)$ it follows that $w_{k}^{ \pm}(y, t ; \tau)=2^{-1 / 2}(1 \mp i t) \varphi_{k}(y), k=l+1, \ldots, m$, and, in the case $\mu<\tau$, the amplitudes of the waves exponentially grow as $t \rightarrow \infty$. The space spanned by the waves (2.10) and (2.11) is called the augmented space of waves for $\tau^{\prime}<\mu<\tau$ and denoted by $W_{a}(\mu)$. Let $W(\mu)$ denote the linear hull of the functions (2.10) and (2.11) for $\tau \leq \mu<\tau^{\prime \prime}$ and the linear hull of the functions (2.11) for $\tau^{\prime}<\mu<\tau$. The lineal $W(\mu)$ is called the space of waves. An element $w \in W_{a}(\mu)($ or $W(\mu))$ is called a wave incoming from $+\infty$ (outgoing to $+\infty)$, if $i q(\chi w, \chi w)<0(i q(\chi w, \chi w)>0)$.

The collection of waves $\left\{w^{ \pm}\right\}_{k=1}^{m}$ defined by (2.10) and (2.11) is called a basis of waves stable in a neighborhood of the threshold $\tau$. A basis of waves of the form (2.6) is by definition stable on $\left(\mu^{\prime}, \mu^{\prime \prime}\right)$ if the interval $\left[\mu^{\prime}, \mu^{\prime \prime}\right]$ contains no thresholds.

\subsection{Waves in the domain $G$}

Let $G$ be a domain in $\mathbb{R}^{n+1}$ with smooth boundary $\partial G$ coinciding, outside a large ball, with the union $\Pi_{+}^{1} \cup \cdots \cup \Pi_{+}^{\mathcal{T}}$ of finitely many nonoverlapping semicylinders

$$
\Pi_{+}^{r}=\left\{\left(y^{r}, t^{r}\right): y^{r} \in \Omega^{r}, t^{r}>0\right\},
$$

where $\left(y^{r}, t^{r}\right)$ are local coordinates in в $\Pi_{+}^{r}$ and $\Omega^{r}$ is a bounded domain in $\mathbb{R}^{n}$.

We introduce the problem

$$
\begin{array}{r}
-\Delta u(x)-\mu u(x)=0, \quad x \in G, \\
u(x)=0, \quad x \in \partial G .
\end{array}
$$

With every $\Pi_{+}^{r}$ we associate a problem of the form (2.1) in the cylinder e $\Pi^{r}=\left\{\left(y^{r}, t^{r}\right)\right.$ : $\left.y^{r} \in \Omega^{r}, t^{r} \in \mathbb{R}\right\}$. Let $\chi \in C^{\infty}(\mathbb{R})$ be a cut-off function, $\chi(t)=0$ for $t<0$ and $\chi(t)=1$ for $t>1$. Each wave in $\Pi^{r}$ we multiply by the function $t \mapsto \chi\left(t^{r}-t_{0}^{r}\right)$ with a certain $t_{0}^{r}>0$ and then extend it by zero to the domain $G$. All functions (for all $\Pi^{r}$ ) obtained in such a way we call waves in $G$. A number $\tau$ is called a threshold for problem (2.17) if the $\tau$ is a threshold at least for one of problems of the form (2.1) in $\Pi^{r}, r=1, \ldots, \mathcal{T}$. Let $\tau^{\prime}<\tau<\tau^{\prime \prime}$ be three successive thresholds for problem $(2.17)$; then the intervals $\left(\tau^{\prime}, \tau\right)$ and $\left(\tau, \tau^{\prime \prime}\right)$ are free from the thresholds.

For $\mu \in\left(\tau^{\prime}, \tau\right)$ we introduce the augmented space $\mathcal{W}_{a}(\mu, G)$ of waves in $G$ as the union of the waves in $G$ corresponding to the waves in $W_{a}(\mu)$ for $\Pi^{r}, r=1, \ldots, \mathcal{T}$; if a space $W_{a}(\mu)$ is not introduced on the interval $\tau^{\prime}<\mu<\tau$ for a certain $\Pi^{r}$ (which means that the $\tau$ is not a threshold for problem (2.1) in such a cylinder), then, from this cylinder, we include into the space $\mathcal{W}_{a}(\mu, G)$ the waves generated by the elements of the corresponding $W(\mu)$. By definition, for $\mu \in\left(\tau^{\prime}, \tau^{\prime \prime}\right)$ the space $\mathcal{W}(\mu, G)$ of waves in $G$ is the union of the waves in $G$ that correspond to the waves in $W(\mu)$ for all $\Pi^{r}$.

The bases $\left\{u_{j}^{ \pm}(\cdot, \mu)\right\}$ and $\left\{w_{j}^{ \pm}(\cdot, \mu)\right\}$ of waves in $\mathcal{W}(\mu, G)$ and $\mathcal{W}_{a}(\mu, G)$ comprise the waves obtained in $G$ from the basis waves in $\Pi^{r}, r=1, \ldots, \mathcal{T}$. The basis waves in the spaces $\mathcal{W}(\mu, G)$ and $\mathcal{W}_{a}(\mu, G)$ are subject to orthogonality and normalization conditions like (2.8) 
and (2.9) or (2.12) and (2.13) with the form q in a cylinder replaced by the form $q_{G}$ in $G$ :

$$
\begin{aligned}
q_{G}(u, v): & =((-\Delta-\mu) u, v)_{G}+\left(u,-\partial_{\nu} v\right)_{\partial G} \\
& -(u,(-\Delta-\mu) v)_{G}-\left(-\partial_{\nu} u, v\right)_{\partial G} .
\end{aligned}
$$

An element $w$ in $\mathcal{W}_{a}(\mu, G)$ (or in $\mathcal{W}(\mu, G)$ ) is called a wave incoming from $\infty$ (outgoing to $\infty)$, if $i q_{G}(\chi w, \chi w)<0\left(i q_{G}(\chi w, \chi w)>0\right)$.

A basis of waves in $G$ is called stable near a value $\nu$ of the spectral parameter if the basis consists of bases in the cylinders $\Pi^{1}, \ldots, \Pi^{\mathcal{T}}$ stable near $\nu$.

\section{Continuous spectrum eigenfunctions. Scattering matrices}

Let $\tau^{\prime}<\tau<\tau^{\prime \prime}$ be three successive thresholds for peoblem (2.17). For the sake of simplicity, we assume that these three numbers are thresholds for a problem of the form (2.1) only in one of the cylinders $\Pi^{1}, \ldots, \Pi^{\mathcal{T}}$, for instance in $\Pi^{1}=\Omega^{1} \times \mathbb{R}$. Moreover, we suppose that $\tau^{\prime}=\mu_{l}, \tau=\mu_{l+1}=\cdots=\mu_{m}$, and $\tau^{\prime \prime}=\mu_{m+1}$, where $\mu_{k}$ are eigenvalues of problem (2.3) in $\Omega^{1}$. Thus for $\Pi=\Pi^{1}$ we deal with the situation considered in 2.1.

\subsection{Intrinsic and expanded radiation principles}

We consider the boundary value problem

$$
\begin{array}{r}
-\Delta u(x)-\mu u(x)=f(x), \quad x \in G, \\
u(x)=g(x), \quad x \in \partial G,
\end{array}
$$

and recall two correct statements of the problem with radiation conditions at infinity: the intrinsic and expanded radiation principles. In the first principle, the intrinsic radiation conditions contain only outgoing waves in the space $\mathcal{W}(\mu, G)$. The second (expanded) principle includes the outgoing waves in the augmented space $\mathcal{W}_{a}(\mu, G)$. For the general elliptic problems self-adjoint with respect to the Green formula, the first statement was discussed in [1] and the second one was considered in [7], [8] (for various geometric situations). We will apply the intrinsic principle with spectral parameter outside a neighborhood of the thresh-

olds. In vicinity of a threshold, we make use of the expanded principle employing the stable basis of waves in $\mathcal{W}_{a}(\mu, G)$ constructed in Section 2.

We first define needed function spaces. For integer $l \geq 0$ we denote by $H^{l}(G)$ the Sobolev space with norm

$$
\left\|v ; H^{l}(G)\right\|=\left(\sum_{j=0}^{l} \int_{G} \sum_{|\alpha|=j}\left|D_{x}^{\alpha} v(x)\right|^{2} d x\right)^{1 / 2},
$$

and let $H^{l-1 / 2}(\partial G)$ with $l \geq 1$ stand for the space of traces on $\partial G$ of the functions in $H^{l}(G)$. Assume that $\rho_{\gamma}$ is a smooth positive on $\bar{G}$ function given on $\Pi_{+}^{r}$ by the equality $\rho_{\gamma}\left(y^{r}, t^{r}\right)=\exp \left(\gamma t^{r}\right)$ with $\gamma \in \mathbb{R}$. We also introduce the spaces $H_{\gamma}^{l}(G)$ and $H_{\gamma}^{l-1 / 2}(\partial G)$ with 
norms $\left\|u ; H_{\gamma}^{l}(G)\right\|=\left\|\rho_{\gamma} u ; H^{l}(G)\right\|$ and $\left\|v ; H_{\gamma}^{l-1 / 2}(\partial G)\right\|=\left\|\rho_{\gamma} v ; H^{l-1 / 2}(\partial G)\right\|$. The operator of problem (3.1) implements the continuous mapping

$$
\mathcal{A}_{\gamma}(\mu): H_{\gamma}^{2}(G) \rightarrow H_{\gamma}^{0}(G) \times H_{\gamma}^{3 / 2}(\partial G) .
$$

As is known, the operator (3.2) is Fredholm if and only if the line $\{\lambda \in \mathbb{C}: \operatorname{Im} \lambda=\gamma\}$ is free of the eigenvalues of the pencils $\lambda \mapsto \mathfrak{A}^{r}(\lambda, \mu), r=1, \ldots, \mathcal{T}$, where $\mathfrak{A}^{r}$ is a pencil of the form (2.2) for the problem (2.1) in the cylinder $\Pi^{r}$. (An operator is called Fredholm, if its range is closed and the kernel and cokernel are finite dimensional.)

We now proceed to the intrinsic radiation principle. Assume that $\mu$ does not coincide with a threshold, $\mu \in\left(\tau^{\prime}, \tau^{\prime \prime}\right)$, and $\mu \neq \tau$. Let $\delta$ denote a small positive number such that the $\operatorname{strip}\{\lambda \in \mathbb{C}:|\operatorname{Im} \lambda| \leq \delta\}$ contains only real eigenvalues of the pencils $\mathfrak{A}^{r}(\cdot, \mu)$, $r=1, \ldots, \mathcal{T}$; we denote the number of such eigenvalues (counted with their multiplicities ) by $2 M=2 M(\mu)$. There exist collections of elements $\left\{Y_{1}^{+}(\cdot, \mu), \ldots, Y_{M}^{+}(\cdot, \mu)\right\}$ and $\left\{Y_{1}^{-}(\cdot, \mu), \ldots, Y_{M}^{-}(\cdot, \mu)\right\}$ in the kernel $\operatorname{ker} \mathcal{A}_{-\delta}(\mu)$ of $\mathcal{A}_{-\delta}(\mu)$ such that

$$
\begin{aligned}
& \left(Y_{j}^{+}(\cdot, \mu)-u_{j}^{+}(\cdot, \mu)-\sum_{k=1}^{M} S_{j k}(\mu) u_{k}^{-}(\cdot, \mu)\right) \in H_{\delta}^{2}(G), \\
& \left(Y_{j}^{-}(\cdot, \mu)-u_{j}^{-}(\cdot, \mu)-\sum_{k=1}^{M} T_{j k}(\mu) u_{k}^{+}(\cdot, \mu)\right) \in H_{\delta}^{2}(G),
\end{aligned}
$$

where $S(\mu)=\left\|S_{j k}(\mu)\right\|$ is a unitary scattering matrix and $S(\mu)^{-1}=T(\mu)=\left\|T_{j k}(\mu)\right\|$. Every collection $\left\{Y_{1}^{+}(\cdot, \mu), \ldots, Y_{M}^{+}(\cdot, \mu)\right\}$ and $\left\{Y_{1}^{-}(\cdot, \mu), \ldots, Y_{M}^{-}(\cdot, \mu)\right\}$ is a basis modulo $\operatorname{ker} \mathcal{A}_{\delta}(\mu)$ in $\operatorname{ker} \mathcal{A}_{-\delta}(\mu)$. This means that any $v \in \operatorname{ker} \mathcal{A}_{-\delta}(\mu)$ is a linear combination of the functions $Y_{1}^{+}(\cdot, \mu), \ldots, Y_{M}^{+}(\cdot, \mu)$ up to a term in $\operatorname{ker} \mathcal{A}_{\delta}(\mu)$; the same is true also for $Y_{1}^{-}(\cdot, \mu), \ldots, Y_{M}^{-}(\cdot, \mu)$. If $\mu$ is not an eigenvalue of operator $(3.2)$, that is, $\operatorname{ker} \mathcal{A}_{\delta}(\mu)=0$, every collection $\left\{Y_{j}^{+}\right\}$and $\left\{Y_{j}^{-}\right\}$is a basis of $\operatorname{ker} \mathcal{A}_{-\delta}(\mu)$ in the usual sense.

The elements $Y(\cdot, \mu)$ in $\operatorname{ker} \mathcal{A}_{-\delta}(\mu) \backslash \operatorname{ker} \mathcal{A}_{\delta}(\mu)$ are called the continuous spectrum eigenfunctions of problem (2.17) corresponding to $\mu$.

Denote by $\mathfrak{N}$ the linear hull $\mathfrak{L}\left(u_{1}^{-}, \ldots, u_{M}^{-}\right)$. We define the norm of $u=\sum c_{j} u_{j}^{-}+v \in$ $\mathfrak{N} \dot{+} H_{\delta}^{2}(G)$ with $c_{j} \in \mathbb{C}$ and $v \in H_{\delta}^{2}(G)$ by

$$
\|u\|=\sum\left|c_{j}\right|+\left\|v ; H_{\delta}^{2}(G)\right\| .
$$

Let $\mathbf{A}(\mu)$ be the restriction of the operator $\mathcal{A}_{-\delta}(\mu)$ to the space $\mathfrak{N} \dot{+} H_{\delta}^{2}(G)$. The map

$$
\mathbf{A}(\mu): \mathfrak{N}+H_{\delta}^{2}(G) \rightarrow H_{\delta}^{0}(G) \times H_{\delta}^{3 / 2}(\partial G)=: \mathcal{H}_{\delta}(G)
$$

is continuous. The next theorem provides the statement of problem (3.1) with intrinsic radiation conditions at infinity (the numbers $\mu$ and $\delta$ are supposed to satisfy the requirements given just before (3.3)).

Theorem 3.1. Let $z_{1}, \ldots, z_{d}$ be a basis in the space $\operatorname{ker} \mathcal{A}_{\delta}(\mu),\{f, g\} \in \mathcal{H}_{\delta}(G)$ and $\left(f, z_{j}\right)_{G}+$ $\left(g,-\partial_{\nu} z_{j}\right)_{\partial G}=0, j=1, \ldots, d$. Then:

1. There exists a solution $u \in \mathfrak{N} \dot{+} H_{\delta}^{2}(G)$ of the equation $\mathbf{A}(\mu) u=\{f, g\}$ determined up to an arbitrary term in $\operatorname{ker} \mathcal{A}_{\delta}(\mu)$. 
2. The inclusion

$$
v \equiv u-c_{1} u_{1}^{-}-\cdots-c_{M} u_{M}^{-} \in H_{\delta}^{2}(G)
$$

holds with $c_{j}=i\left(f, Y_{j}^{-}\right)_{G}+i\left(g,-\partial_{\nu} Y_{j}^{-}\right)_{\partial G}$.

3. The inequality

$$
\left\|v ; H_{\delta}^{2}(G)\right\|+\left|c_{1}\right|+\cdots+\left|c_{M}\right| \leq \operatorname{const}\left(\left\|\{f, g\} ; \mathcal{H}_{\delta}(G)\right\|+\left\|\rho_{\delta} v ; L_{2}(G)\right\|\right) .
$$

holds with $v$ and $c_{1}, \ldots, c_{M}$ in (3.6). A solution $u_{0}$ that is subject to the additional conditions $\left(u_{0}, z_{j}\right)_{G}=0$ for $j=1, \ldots, d$ is unique and satisfies (3.7) with right-hand side changed for const $\left\|\{f, g\} ; \mathcal{H}_{\delta}(G)\right\|$.

4. If $\{f, g\} \in \mathcal{H}_{\delta}(G) \cap \mathcal{H}_{\delta^{\prime}}(G)$ and the strip $\left\{\lambda \in \mathbb{C}: \min \left\{\delta, \delta^{\prime}\right\} \leq \operatorname{Im} \lambda \leq \max \left\{\delta, \delta^{\prime}\right\}\right\}$ contains no eigenvalues of the pencils $\mathfrak{A}^{r}(\cdot, \mu), r=1, \ldots, \mathcal{T}$, then the solutions $u \in \mathfrak{N} \dot{+}$ $H_{\delta}^{2}(G)$ and $u^{\prime} \in \mathfrak{N}+H_{\delta^{\prime}}^{2}(G)$ coincide, while the choice between $\delta$ and $\delta^{\prime}$ in essence effects only the constant in (3.7).

Remark 3.2. In Theorem 3.1, one can take the numbers $\delta$ and "const" in (3.7) invariant for all $\mu$ in $\left[\mu^{\prime}, \mu^{\prime \prime}\right] \subset\left(\tau, \tau^{\prime \prime}\right)$ (in $\left.\left[\mu^{\prime}, \mu^{\prime \prime}\right] \subset\left(\tau^{\prime}, \tau\right)\right)$. If $\mu^{\prime \prime}$ approaches $\tau^{\prime \prime}(\tau)$, the $\delta$ must tend to zero: an admissible interval for $\delta$ has to be narrowed because the imaginary eigenvalues of the pencils move closer to the real axis; the constant in (3.7) increases. From the proof of Theorem 3.1 in [1] one can see that the constant also increases when $\mu^{\prime}$ approaches $\tau$ (or $\left.\tau^{\prime}\right)$.

We now turn to the expanded radiation principle in a neihgborhood of $\tau$. To this end, for problem (2.17), we construct a basis of waves stable at the threshold $\tau$. We make up such a basis from the waves generated by the functions (2.10), (2.11) and from the waves corresponding to the real eigenvalues of the pencils $\mathfrak{A}^{r}(\cdot, \mu), r=2, \ldots, \mathcal{T}$. According to our assumption (at the beginning of Section 3), the interval $\left[\tau^{\prime}, \tau^{\prime \prime}\right]$ contains no threshold for problems of the form $(2.1)$ in the cylinders $\Pi^{2}, \ldots, \Pi^{\mathcal{T}}$. Therefore the number of real eigenvalues for every of the pencils $\mathbb{R} \ni \lambda \rightarrow \mathfrak{A}^{r}(\lambda, \mu), r=2, \ldots, \mathcal{T}$, remains invariant for $\mu \in\left[\tau^{\prime}, \tau^{\prime \prime}\right]$. Thus when passing from the cylinder $\Pi^{1}$ to the domain $G$, the dimension of wave space increases by the same number for all $\mu \in\left(\tau^{\prime}, \tau^{\prime \prime}\right)$. We set $2 L=\operatorname{dim} \mathcal{W}(\mu, G)$ for $\mu \in\left(\tau^{\prime}, \tau\right)$ and $2 M=\operatorname{dim} \mathcal{W}(\mu, G)$ for $\mu \in\left(\tau, \tau^{\prime \prime}\right)$; then $M-L=m-l$, where $m$ and $l$ are the same as in (2.10), (2.11), and $\operatorname{dim} \mathcal{W}_{a}(\mu, G)=2 M$ for $\mu \in\left(\tau^{\prime}, \tau\right)$.

We choose the number $\gamma$ for the operators $\mathcal{A}_{ \pm \gamma}(\mu)$ to be proper for all $\mu$ in a neighborhood of the threshold $\tau=\mu_{m}$. Let us explain such a choice. We have $\lambda_{k}^{ \pm}(\mu)= \pm\left(\mu-\mu_{k}\right)^{1 / 2}$, $\mu_{l+1}=\ldots, \mu_{m}$, so $\lambda_{k}^{ \pm}(\tau)=0$ with $k=l+1, \ldots, m$. The interval of the imaginary axis with ends $-i\left(\mu_{m+1}-\mu_{m}\right)^{1 / 2}, i\left(\mu_{m+1}-\mu_{m}\right)^{1 / 2}$ punctured at the coordinate origin is free of the spectra of the pencils $\mathfrak{A}^{q}\left(\cdot, \mu_{m}\right), q=1, \ldots, \mathcal{T}$. If $\mu$ a little moves along $\mathbb{R}$, the eigenvalues of the pencils $\mathfrak{A}^{q}(\cdot, \mu)$ slightly shift along the coordinate axes. Therefore, for a small $\alpha>0$ there exists $\beta>0$ such that for $\mu \in\left(\mu_{m}-\beta, \mu_{m}+\beta\right)$ the intervals $i I_{ \pm \alpha}:= \pm i\left(\alpha,\left(\mu_{m+1}-\mu_{m}\right)^{1 / 2}-\alpha\right)$ are free of the spectra of the pencils $\mathfrak{A}^{q}(\cdot, \mu)$. So the lines $\{\lambda \in \mathbb{C}: \operatorname{Im} \lambda= \pm \gamma\}$ with $\gamma \in I_{\alpha}$ do not intersect the spectra of $\mathfrak{A}^{q}(\cdot, \mu)$, while the strip $\{\lambda \in \mathbb{C}:|\operatorname{Im} \lambda| \leq \gamma\}$ contains only the real eigenvalues of the pencils and the numbers $\lambda_{k}^{ \pm}(\mu)= \pm\left(\mu-\mu_{k}\right)^{1 / 2}= \pm\left(\mu-\mu_{m}\right)^{1 / 2}$ in $(2.10), k=l+1, \ldots, m$. 
Let $\mu \in(\tau-\beta, \tau+\beta), \gamma \in I_{\alpha}$, and let $\left\{w_{k}^{ \pm}(\cdot, \mu)\right\}$ be the stable basis of waves in $G$ described in 2.1 and 2.2. In the kernel $\operatorname{ker} \mathcal{A}_{-\gamma}(\mu)$ of $\mathcal{A}_{-\gamma}(\mu)$, there exist collections of elements $\left\{\mathcal{Y}_{1}^{+}(\cdot, \mu), \ldots, \mathcal{Y}_{M}^{+}(\cdot, \mu)\right\}$ and $\left\{\mathcal{Y}_{1}^{-}(\cdot, \mu), \ldots, \mathcal{Y}_{M}^{-}(\cdot, \mu)\right\}$ such that

$$
\begin{aligned}
& \left(\mathcal{Y}_{j}^{+}(\cdot, \mu)-w_{j}^{+}(\cdot, \mu)-\sum_{k=1}^{M} \mathcal{S}_{j k}(\mu) w_{k}^{-}(\cdot, \mu)\right) \in H_{\gamma}^{2}(G), \\
& \left(\mathcal{Y}_{j}^{-}(\cdot, \mu)-w_{j}^{-}(\cdot, \mu)-\sum_{k=1}^{M} \mathcal{T}_{j k}(\mu) w_{k}^{+}(\cdot, \mu)\right) \in H_{\gamma}^{2}(G),
\end{aligned}
$$

where $\mathcal{S}(\mu)=\left\|\mathcal{S}_{j k}(\mu)\right\|$ is the unitary matrix and $\mathcal{S}(\mu)^{-1}=\mathcal{T}(\mu)=\left\|\mathcal{T}_{j k}(\mu)\right\|$. Every collection $\left\{\mathcal{Y}_{1}^{+}(\cdot, \mu), \ldots, \mathcal{Y}_{M}^{+}(\cdot, \mu)\right\}$ and $\left\{\mathcal{Y}_{1}^{-}(\cdot, \mu), \ldots, \mathcal{Y}_{M}^{-}(\cdot, \mu)\right\}$ is a basis (modulo ker $\mathcal{A}_{\gamma}(\mu)$ ) in $\operatorname{ker} \mathcal{A}_{-\gamma}(\mu)$.

The elements $\mathcal{Y}(\cdot, \mu)$ in $\operatorname{ker} \mathcal{A}_{-\gamma}(\mu) \backslash \operatorname{ker} \mathcal{A}_{\gamma}(\mu)$ are called the continuous spectrum eigenfunctions of problem (2.17) corresponding to the number $\mu$. The matrix $\mathcal{S}(\mu)$ (with $\mu \in(\tau-\beta, \tau))$ is called the augmented scattering matrix.

Let $\mathfrak{K}$ denote the linear hull $\mathfrak{L}\left(w_{1}^{-}, \ldots, w_{M}^{-}\right)$. We define a norm of $w=\sum c_{j} w_{j}^{-}+v \in$ $\mathfrak{K} \dot{+} H_{\gamma}^{2}(G)$, where $c_{j} \in \mathbb{C}$ and $v \in H_{\gamma}^{2}(G)$, by the equality

$$
\|w\|=\sum\left|c_{j}\right|+\left\|v ; H_{\gamma}^{2}(G)\right\|
$$

Let $\mathbf{A}(\mu)$ be the restriction of $\mathcal{A}_{-\gamma}(\mu)$ to the space $\mathfrak{K} \dot{+} H_{\gamma}^{2}(G)$; then the mapping

$$
\mathbf{A}(\mu): \mathfrak{K} \dot{+} H_{\gamma}^{2}(G) \rightarrow H_{\gamma}^{0}(G) \times H_{\gamma}^{3 / 2}(\partial G)=: \mathcal{H}_{\gamma}(G) .
$$

is continuous.

Theorem 3.3. Let $\mu \in(\tau-\beta, \tau+\beta), \gamma \in I_{\alpha}$, and let $\left\{w_{k}^{ \pm}(\cdot, \mu)\right\}$ be the aforementioned stable basis of waves in $G$. Assume $z_{1}, \ldots, z_{d}$ to be a basis in the space $\operatorname{ker} \mathcal{A}_{\gamma}(\mu),\{f, g\} \in \mathcal{H}_{\gamma}(G)$ and $\left(f, z_{j}\right)_{G}+\left(g,-\partial_{\nu} z_{j}\right)_{\partial G}=0, j=1, \ldots, d$. Then:

1). There exists a solution $w \in \mathfrak{K} \dot{+} H_{\gamma}^{2}(G)$ to the equation $\mathbf{A}(\mu) w=\{f, g\}$ determined up to an arbitrary term in the lineal $\mathcal{L}\left(z_{1}, \ldots, z_{d}\right)$.

2). The inclusion

$$
v \equiv w-c_{1} w_{1}^{-}-\cdots-c_{M} w_{M}^{-} \in H_{\gamma}^{2}(G)
$$

holds with $c_{j}=i\left(f, \mathcal{Y}_{j}^{-}\right)_{G}+i\left(g,-\partial_{\nu} \mathcal{Y}_{j}^{-}\right)_{\partial G}$.

3). Such a solution $w$ satisfies the inequality

$$
\left\|v ; H_{\gamma}^{2}(G)\right\|+\left|c_{1}\right|+\cdots+\left|c_{M}\right| \leq \operatorname{const}\left(\left\|\{f, g\} ; \mathcal{H}_{\gamma}(G)\right\|+\left\|\rho_{\gamma} v ; L_{2}(G)\right\|\right) .
$$

$A$ solution $w_{0}$ that is subject to the conditions $\left(w_{0}, z_{j}\right)_{G}=0$ for $j=1, \ldots, d$ is unique and the estimate (3.12) holds with the right-hand side changed for const $\left\|\{f, g\} ; \mathcal{H}_{\gamma}(G)\right\|$.

4). If $\{f, g\} \in \mathcal{H}_{\gamma}(G) \cap \mathcal{H}_{\gamma^{\prime}}(G)$ and the strip $\left\{\lambda \in \mathbb{C}: \min \left\{\gamma, \gamma^{\prime}\right\} \leq \operatorname{Im} \lambda \leq\right.$ $\left.\max \left\{\gamma, \gamma^{\prime}\right\}\right\}$ contains no eigenvalues of the pencils $\mathfrak{A}^{r}(\cdot, \mu), r=1, \ldots, \mathcal{T}$, the solutions $w(\cdot, \mu) \in \mathfrak{K}+H_{\gamma}^{2}(G)$ and $w^{\prime}(\cdot, \mu) \in \mathfrak{K} \dot{+} H_{\gamma^{\prime}}^{2}(G)$ of the equation $\mathbf{A}(\mu) w=\{f, g\}$ coincide, while the choice between $\gamma$ and $\gamma^{\prime}$, in essence, effects only the constant in (3.12). 
We would like to extend relations of the form (3.8) and (3.9) to the interval $\left(\tau^{\prime}, \tau^{\prime \prime}\right)$ with analytic functions $\mu \mapsto \mathcal{Y}_{j}^{ \pm}(\mu)$. Unlike the situation in Remark 3.2, it is not possible, generally speaking, to extend (3.8) and (3.9) to any interval $\left[\mu^{\prime}, \mu^{\prime \prime}\right] \subset\left(\tau^{\prime}, \tau^{\prime \prime}\right)$ with the same index $\gamma$. However, to that purpose, one can use a finite collection of indices for various parts of $\left[\mu^{\prime}, \mu^{\prime \prime}\right]$. The following lemma explains how to compile such a collection.

Lemma 3.4. For any interval $\left[\mu^{\prime}, \mu^{\prime \prime}\right] \subset\left(\tau^{\prime}, \tau^{\prime \prime}\right)$ there exists a finite covering $\left\{U_{p}\right\}_{p=0}^{N}$ consisting of open intervals and a collection of indices $\left\{\gamma^{p}\right\}_{p=0}^{N}$ subject to the following conditions (with a certain nonnegative number $N$ ):

1) $\mu^{\prime} \in U_{0}, \mu^{\prime \prime} \in U_{N} ; U_{0} \cap U_{p}=\emptyset, \quad p=2, \ldots, N ; \quad U_{N} \cap U_{p}=\emptyset, \quad p=0, \ldots, N-$ 2; moreover, $U_{p}$ only overlaps $U_{p-1}$ and $U_{p+1}, 1 \leq p \leq N-1$.

2) The line $\left\{\lambda \in \mathbb{C}: \operatorname{Im} \lambda=\gamma^{p}\right\}$ is free from the spectra of the pencils $\mathfrak{A}^{r}(\cdot, \mu)$, $r=1, \ldots, \mathcal{T}$, for all $\mu \in U_{p} \cap\left[\mu^{\prime}, \mu^{\prime \prime}\right]$ and $p=0, \ldots, N$.

3) The strip $\left\{\lambda \in \mathbb{C}: \gamma^{p} \leq \operatorname{Im} \lambda \leq \gamma^{p+1}\right\}$ is free from the spectra of the pencils $\mathfrak{A}^{r}(\cdot, \mu)$, $r=1, \ldots, \mathcal{T}$ for all $\mu \in U_{p} \cap U_{p+1}$ and $p=0, \ldots, N-1$.

4) The inequality $\left|\operatorname{Im}(\mu-\tau)^{1 / 2}\right|<\gamma^{p}$ holds for $\mu \in U_{p} \cap\left[\mu^{\prime}, \mu^{\prime \prime}\right]\left(\right.$ recall that $\pm(\mu-\tau)^{1 / 2}$ are eigenvalues of $\left.\mathfrak{A}^{1}(\cdot, \mu), \tau=\mu_{l+1}=\cdots=\mu_{m}\right)$; there are $n o$ other eigenvalues of the pencils $\mathfrak{A}^{r}(\cdot, \mu), r=1, \ldots, \mathcal{T}$, in the strip $\left\{\lambda \in \mathbb{C}:|\operatorname{Im} \lambda| \leq \gamma^{p}\right\}$ except the real ones, $p=0, \ldots, N$.

Proof. We outline the proof. Let us consider an interval $\left[\mu^{\prime}, \mu^{\prime \prime}\right]$ assuming $\tau \in\left(\mu^{\prime}, \mu^{\prime \prime}\right)$. Just before formulas (3.8) and (3.9), we have defined the interval $(\tau-\beta, \tau+\beta)$ that can be taken as an element of the desired covering. It was earlier shown that as an index $\gamma$ for such an element one can choose any number in $I_{\alpha}=\left(\alpha,\left(\mu_{m+1}-\mu_{m}\right)^{1 / 2}-\alpha\right)$ with small positive $\alpha$; the number $\beta$ depends on $\alpha$.

Let us take some $\nu \in(\tau, \tau+\beta)$. The eigenvalue $\lambda_{m}(\mu)=\left(\mu-\mu_{m}\right)^{1 / 2}$ of the pencil $\mathfrak{A}^{1}(\cdot, \mu)$ is real for $\mu>\nu$, the eigenvalue $\lambda_{m+1}(\mu)=i\left(\mu_{m+1}-\mu\right)^{1 / 2}$ of the pencil tends to zero when $\mu$ increases from $\nu$ to $\tau^{\prime \prime}=\mu_{m+1}$, and the interval $\{z \in \mathbb{C}: z=i t, 0<t<$ $\left.\left(\mu_{m+1}-\mu^{\prime \prime}\right)^{1 / 2}\right\}$ of the imaginary axis remains free from the spectra of the pencils $\mathfrak{A}^{r}(\cdot, \mu)$, $\mu^{\prime} \leq \mu \leq \mu^{\prime \prime}, r=1, \ldots, \mathcal{T}$. Therefore the interval $(\nu, \tilde{\nu})$ with $\mu^{\prime \prime}<\tilde{\nu}<\tau^{\prime \prime}$ can serve as an element of the covering and any number $\gamma \in\left(0,\left(\mu_{m+1}-\mu^{\prime \prime}\right)^{1 / 2}\right)$ can be an index for the element. Finally we choose the elements $U_{p}$ to the left of the threshold $\tau$ so that the graphs of the functions $U_{p} \ni \mu \mapsto \gamma^{p}=$ const are located between the graphs of the functions $\left(\tau^{\prime}, \tau\right) \ni \mu \mapsto \operatorname{Im} \lambda_{k}(\mu)=\left(\mu_{k}-\mu\right)^{1 / 2}, k=m, m+1$, and the indices form a decreasing sequence $\gamma^{0}>\gamma^{1}>\ldots$.

\subsection{Analyticity of scattering matrices with respect to spectral parameter}

Let us consider the bases $\left\{\mathcal{Y}_{j}^{+}\right\}$and $\left\{\mathcal{Y}_{j}^{-}\right\}$in the spaces of continuous spectrum eigenfunctions (CSE) defined near the threshold $\tau$ (see (3.8) and (3.9)). We first show that the functions $\mu \mapsto \mathcal{Y}_{j}^{ \pm}(\cdot, \mu)$ admit the analytic extension to the interval $\left(\tau^{\prime}, \tau^{\prime \prime}\right)$. In what follows by the analyticity of a function on an interval we mean the possibility of analytic continuation of the function in a complex neighborhood of every point in the interval. Then we prove the analyticity of the scattering matrix $\mu \mapsto \mathcal{S}(\mu)$ on $\left(\tau^{\prime}, \tau^{\prime \prime}\right)$. The analyticity does not exclude the existence of eigenvalues of problem (2.17) embedded into the continuous spectrum; however, the analyticity eliminates the arbitrariness in the choice of CSE. Moreover, we establish the 
analyticity of the elements $\mu \mapsto Y_{j}^{ \pm}(\cdot, \mu)$ in (3.3) and (3.4) as well as the analyticity of the corresponding scattering matrix $\mu \mapsto S(\mu)$ on $\left(\tau^{\prime}, \tau\right)$ and $\left(\tau, \tau^{\prime \prime}\right)$.

In a neighborhood of any point of the interval $\left(\tau^{\prime}, \tau^{\prime \prime}\right)$, one can define an operator $\mathcal{A}_{\gamma}(\mu)$, which is needed for relations like (3.8) and (3.9). The index $\gamma$ has been provided by Lemma 3.4: the same number $\gamma^{p}$ can serve for all $\mu \in U_{p}$. Therefore, for $\mu \in U_{p}$ there exist the families $\left\{\mathcal{Y}_{j}^{ \pm}(\cdot, \mu)\right\} \subset \operatorname{ker} \mathcal{A}_{-\gamma^{p}}(\mu)$ satisfying relations like (3.8) and (3.9) with unitary matrix $\mathcal{S}(\mu)$, so Theorem 3.3 holds with $\mu \in U_{p}$. Thus, it suffices to prove the analyticity of the "local families" $\left\{\mathcal{Y}_{j}^{ \pm}(\cdot, \mu)\right\}$ and that of the matrix $\mathcal{S}(\mu)$ on $U_{p}$ and to verify the compatibility of such families on the intersections of neighborhoods.

We first obtain a representation of the operator $\mathbf{A}(\mu)^{-1}$, where $\mathbf{A}(\mu)$ is operator (3.5) or (3.10), in a neighborhood of an eigenvalue of problem (2.17). To this end we recall some facts in the theory of holomorphic operator-valued functions (e. g., see [9]). Let $\mathcal{D}$ be a domain in a complex plane, $B_{1}$ and $B_{2}$ Banach spaces, and $\mathbb{A}$ a holomorphic operator-valued function $\mathcal{D} \ni \mu \mapsto \mathbb{A}(\mu): B_{1} \rightarrow B_{2}$. The spectrum of the function $\mathbb{A}(\cdot)$ is the set of points $\mu \in \mathcal{D}$ such that $\mathbb{A}(\mu)$ is a noninvertible operator. A number $\mu_{0}$ is called an eigenvalue of $\mathbb{A}$ if there exists a nonzero vector $\varphi_{0} \in B_{1}$ such that $\mathbb{A}\left(\mu_{0}\right) \varphi_{0}=0$; then $\varphi_{0}$ is called an eigenvector. Let $\mu_{0}$ and $\varphi_{0}$ be an eigenvalue and an eigenvector. Elements $\varphi_{1}, \ldots, \varphi_{m-1}$ are called generalized eigenvectors, if

$$
\sum_{q=0}^{n} \frac{1}{q !}\left(\partial_{\mu}^{q} \mathbb{A}\right)\left(\mu_{0}\right) \varphi_{n-q}=0,
$$

where $n=1, \ldots, m$. A holomorphic function $\mathbb{A}$ is said to be Fredholm, if the operator $\mathbb{A}(\mu): B_{1} \rightarrow B_{2}$ is Fredholm for all $\mu \in \mathcal{D}$ and is invertible at least for one $\mu$. The spectrum of a Fredholm function $\mathbb{A}$ consists of isolated eigenvalues of finite algebraic multiplicity. The holomorphic function $\mathbb{A}^{*}$ adjoint to $\mathbb{A}$ is defined on the set $\{\mu: \bar{\mu} \in \mathcal{D}\}$ by the equality $\mathbb{A}^{*}(\mu)=(\mathbb{A}(\bar{\mu}))^{*}: B_{1}^{*} \rightarrow B_{2}^{*}$. If one of the functions $\mathbb{A}$ and $\mathbb{A}^{*}$ is Fredholm, then the other one is also Fredholm. A number $\mu_{0}$ is an eigenvalue of $\mathbb{A}$ if and only if $\bar{\mu}_{0}$ is an eigenvalue of $\mathbb{A}^{*}$; the algebraic and geometric multipicities of $\bar{\mu}_{0}$ coincide with those of $\mu_{0}$.

Let us consider the operator-valued function $\mu \mapsto \mathbf{A}(\mu)$ in (3.5) or (3.10) on an interval $\left[\mu^{\prime}, \mu^{\prime \prime}\right]$ that belongs to one of the intervals $\left(\tau^{\prime}, \tau\right)$ or $\left(\tau, \tau^{\prime \prime}\right)$. Taking account of Remark 3.2, we choose the same index $\delta$ in (3.5) and in Theorem 3.1 for all $\mu \in\left[\mu^{\prime}, \mu^{\prime \prime}\right]$. When considering the function $\mu \mapsto \mathbf{A}(\mu)$ in (3.10) on an interval $\left[\mu^{\prime}, \mu^{\prime \prime}\right] \subset\left(\tau^{\prime}, \tau^{\prime \prime}\right)$, we suppose the interval to be so small that Lemma 3.4 enables us to take the same $\gamma$ in (3.10) and in Theorem 3.3 for all $\mu \in\left[\mu^{\prime}, \mu^{\prime \prime}\right]$. According to Proposition 2.1, the waves in the definitions of operators (3.5) and (3.10) are holomorphic in a complex neighborhood of the corresponding interval $\left[\mu^{\prime}, \mu^{\prime \prime}\right]$. Therefore, the functions $\mu \mapsto \mathbf{A}(\mu)$ in Theorems 3.1 and 3.3 are holomorphic in the same neighborhood.

Proposition 3.5. i). Let $\mu \mapsto \mathbf{A}(\mu)$ be the function in Theorem 3.3, $\mu_{0}$ an eigenvalue of operator (3.2), and $\left(z_{1}, \ldots, z_{d}\right)$ a basis of $\operatorname{ker} \mathcal{A}_{\gamma}\left(\mu_{0}\right)$. Then in a punctured neighborhood of $\mu_{0}$ there holds the representation

$$
\mathbf{A}^{-1}(\mu)\{f, g\}=\left(\mu-\mu_{0}\right)^{-1} \mathbf{P}\{f, g\}+\mathbf{R}(\mu)\{f, g\},
$$


where $\{f, g\} \in \mathcal{H}_{\gamma}(G)$,

$$
\mathbf{P}\{f, g\}=-\sum_{j=1}^{d}\left(\left(f, z_{j}\right)_{G}+\left(g,-\partial_{\nu} z_{j}\right)_{\partial G}\right) z_{j},
$$

and the function $\mathbf{R}(\mu): \mathcal{H}_{\gamma}(G) \rightarrow \mathfrak{K}+H_{\gamma}^{2}(G)$ is holomorphic in a neighborhood of $\mu_{0}$.

ii). Let $\mu \mapsto \mathbf{A}(\mu)$ be the operator-valued function in Theorem 3.1, $\mu_{0}$ an eigenvalue of operator $(3.2)$ in $\left(\tau^{\prime}, \tau\right)$ or $\left(\tau, \tau^{\prime \prime}\right)$, and $\left(z_{1}, \ldots, z_{d}\right)$ a basis of $\operatorname{ker} \mathcal{A}_{\delta}\left(\mu_{0}\right)$. Then in a punctured neighborhood of $\mu_{0}$ there holds representation (3.13), where $\mathbf{P}\{f, g\}$ is defined by (3.14) and the function $\mathbf{R}(\mu): \mathcal{H}_{\delta}(G) \rightarrow \mathfrak{N}+H_{\delta}^{2}(G)$ is holomorphic in a neighborhood of $\mu_{0}$.

Proof. i). By Theorem $3.3,1)$, the operator $\mathbf{A}(\mu)$ is Fredholm at any $\mu \in\left[\mu_{m}^{\prime}, \mu_{m}^{\prime \prime}\right]$. We can consider that $\mathbf{A}(\mu)$ is Fredholm in a neighborhood $U$ (the Fredholm property is stable with respect to perturbations that are small in the operator norm). Moreover, the operator $\mathbf{A}(\mu)$ is invertible for all $\mu \in\left[\mu_{m}^{\prime}, \mu_{m}^{\prime \prime}\right]$ except the eigenvalues of operator (3.2), which are real and isolated. Hence the function $\mu \mapsto \mathbf{A}(\mu)$ is Fredholm in a neighborhood of $\mu_{0}$ in the complex plane. From Theorem 3.3,4), it follows that the eigenspaces of operators (3.10) and (3.2) coincide, that is, $\operatorname{ker} \mathbf{A}\left(\mu_{0}\right)=\operatorname{ker} \mathcal{A}_{\gamma}\left(\mu_{0}\right) \subset H_{\gamma}^{2}(G)$. It is easy to verify that the operatorvalued function $\mathbf{A}$ has no generalized eigenvectors at $\mu_{0}$. Then the Keldysh theorem on the resolvent of holomorphic operator-valued fuction (see [9]) provides the equality

$$
\mathbf{A}^{-1}(\mu)\{f, g\}=\left(\mu-\mu_{0}\right)^{-1} \mathbf{T}\{f, g\}+\mathbf{R}(\mu)\{f, g\} ;
$$

here $\mathbf{T}\{f, g\}=\sum_{j=1}^{d}\left\langle\{f, g\},\left\{\psi_{j}, \chi_{j}\right\}\right\rangle z_{j}$, the duaity $\langle\cdot, \cdot\rangle$ on the pair $\mathcal{H}_{\gamma}(G), \mathcal{H}_{\gamma}(G)^{*}$ is defined by $\langle\{f, g\},\{\psi, \chi\}\rangle=(f, \psi)_{G}+(g, \chi)_{\partial G}$, and $(\cdot, \cdot)_{G}$ and $(\cdot, \cdot)_{\partial G}$ are the extensions of the inner products on $L_{2}(G)$ and $L_{2}(\partial G)$ to the pairs $H_{\gamma}^{0}(G), H_{\gamma}^{0}(G)^{*}$ and $H_{\gamma}^{3 / 2}(\partial G), H_{\gamma}^{3 / 2}(\partial G)^{*}$, respectively. The elements $\left\{\psi_{j}, \chi_{j}\right\} \in \operatorname{ker} \mathbf{A}\left(\mu_{0}\right)^{*} \subset W(G ; \gamma)^{*}$ are subject to the orthogonality and normalization conditions

$$
\left\langle\left(\partial_{\mu} \mathbf{A}\right)\left(\mu_{0}\right) z_{j},\left\{\psi_{k}, \chi_{k}\right\}\right\rangle=\delta_{j k}, j, k=1, \ldots, d .
$$

Furthermore, $\left(\partial_{\mu} \mathbf{A}\right)\left(\mu_{0}\right) z_{j}=\left\{-z_{j}, 0\right\} \in W(G ; \gamma)$. The elements $\left\{\psi_{k}, \chi_{k}\right\}$ can be interpreted in terms of the Green formula and,in view of (3.16), rewritten in the form $\left\{\psi_{k}, \chi_{k}\right\}=$ $\left\{-z_{k}, \partial_{\nu} z_{k}\right\}$ (e.g., see [1]). Now $\mathbf{T}\{f, g\}$ coincides with $\mathbf{P}\{f, g\}$ in (3.14) and (3.15) takes the form of (3.13).

ii). One can repeat with evident modifications the argument in (i).

We are now ready to discuss the analyticity of bases in the space of the continuous spectrum eigenfunctions. For instance, we proceed to the basis $\left\{\mathcal{Y}_{j}^{+}\right\}$in (3.8). From the definition of the wave $w_{j}^{+}$in $G$ (see 2.2), it follows that the function $G \ni x \mapsto w_{j}^{+}(x, \mu)$ is supported by one of the cylindrical ends of $G$,

$$
\begin{array}{r}
-\Delta w_{j}^{+}(x, \mu)-\mu w_{j}^{+}(x, \mu)=f_{j}(x, \mu), \quad x \in G, \\
w_{j}^{+}(x, \mu)=0, \quad x \in \partial G,
\end{array}
$$

and the support of the function $x \mapsto f_{j}(x, \mu)$ is compact. Let us consider the equation

$$
\mathbf{A}(\mu) w(\cdot, \mu)=\left\{f_{j}(\cdot, \mu), 0\right\}
$$


on an interval $\left[\mu^{\prime}, \mu^{\prime \prime}\right] \subset\left(\tau^{\prime}, \tau^{\prime \prime}\right)$. We first assume that the interval $\left[\mu^{\prime}, \mu^{\prime \prime}\right]$ is free of the eigenvalues of the operator-valued function $\mu \mapsto \mathbf{A}(\mu)$. In view of Theorem 3.3, for all $\mu \in\left[\mu^{\prime}, \mu^{\prime \prime}\right]$ there exists a unique solution $w=v+c_{1} w_{1}^{-}+\cdots+c_{M} w_{M}^{-}$to equation (3.17),

$$
w(\cdot, \mu)=\left\{c_{1}(\mu), \ldots, c_{M}(\mu), v(\cdot, \mu)\right\} \in \mathfrak{K} \dot{+} H_{\gamma}^{2}(G) .
$$

Since the functions $\mu \mapsto \mathbf{A}(\mu)^{-\mathbf{1}}$ and $\mu \mapsto f_{j}(\cdot, \mu)$ are holomorphic in a complex neighborhood of the interval $\left[\mu^{\prime}, \mu^{\prime \prime}\right]$, the components of the vector-valued function $\mu \mapsto w(\cdot, \mu)$ are holomorphic as well. Therefore, the analyticity of the function $\mu \mapsto \mathcal{Y}_{j}^{+}(\cdot, \mu)$ in the same neighborhood follows from the equality

$$
\mathcal{Y}_{j}^{+}=w_{j}^{+}-w
$$

Assume now that the interval $\left[\mu^{\prime}, \mu^{\prime \prime}\right]$ contains an eigenvalue $\mu_{0}$ of the operator-valued function $\mu \mapsto \mathbf{A}(\mu)$. We find the residue $\mathbf{P}\{f, g\}$ in (3.13) for $\{f, g\}=\left\{f_{j}, 0\right\}$ in the right-hand side of (3.17). For $z \in \operatorname{ker} \mathcal{A}_{\gamma}\left(\mu_{0}\right)$, we have

$$
(f, z)_{G}+\left(g,-\partial_{\nu} z\right)_{\partial G}=\left(f_{j}, z\right)_{G}=\left(-\Delta w_{j}^{+}-\mu w_{j}^{+}, z\right)_{G}=\left(w_{j}^{+},-\Delta z-\mu z\right)_{G}=0 .
$$

Hence $\mathbf{P}\left\{f_{j}, 0\right\}=0$ and, by virtue of (3.13),

$$
w(\cdot, \mu)=\mathbf{A}(\mu)^{-1}\left\{f_{j}, 0\right\}=\mathbf{R}(\mu)\left\{f_{j}, 0\right\},
$$

which means that the function $\mu \mapsto w(\cdot, \mu)$ is analytic in a neighborhood of $\mu_{0}$. This implies the analyticity of the function $\mu \mapsto \mathcal{Y}_{j}^{+}(\cdot, \mu)$.

The analyticity of the functions $\mu \mapsto \mathcal{Y}_{j}^{-}(\cdot, \mu)$ can be proved in the same way. When verifying the analyticity of functions of the form $\mu \mapsto Y_{j}^{+}(\cdot, \mu)$ and $\mu \mapsto Y_{j}^{-}(\cdot, \mu)$ in $(3.3)$ and (3.4) in a complex neighborhood of the interval $\left[\mu^{\prime}, \mu^{\prime \prime}\right] \subset\left(\tau^{\prime}, \tau\right)$ or $\left[\mu^{\prime}, \mu^{\prime \prime}\right] \subset\left(\tau, \tau^{\prime \prime}\right)$, one has to make only evident modification of the above argument.

Lemma 3.4 and Theorem 3.3, 4) enable us to extend formulas (3.8) and (3.9) to the whole interval $\left(\tau^{\prime}, \tau^{\prime \prime}\right)$ for the analytic families $\mu \mapsto \mathcal{Y}_{j}^{ \pm}(\cdot, \mu)$; however, one index $\gamma$ has to be replaced by a collection of indices. Nontheless, in a neighdorhood of any given point $\mu \in\left(\tau^{\prime}, \tau^{\prime \prime}\right)$, one can do with one index $\gamma$. Remark 3.2 and Theorem 3.1,4) allow to extend (3.3) and (3.4) to the intervals $\left(\tau^{\prime}, \tau\right)$ and $\left(\tau, \tau^{\prime \prime}\right)$ for the analytic families $\mu \mapsto Y_{j}^{ \pm}(\cdot, \mu)$.

Theorem 3.6. Let $\tau^{\prime}$ and $\tau^{\prime \prime}$ be thresholds of problem (2.17) such that $\tau^{\prime}<\tau^{\prime \prime}$ and the interval $\left(\tau^{\prime}, \tau^{\prime \prime}\right)$ contains the only threshold $\tau$. We also suppose that the three thresholds relate to the same cylindrical end. Then:

i). On the intervals $\left(\tau^{\prime}, \tau\right)$ and $\left(\tau, \tau^{\prime \prime}\right)$, there exist analytic bases $\left\{\mu \mapsto Y_{j}^{ \pm}(\cdot, \mu)\right\}$ in the spaces of continuous spectrum eigenfunctions of problem (2.17) satisfying (3.3) and (3.4) with the scattering matrix $\mu \mapsto S(\mu)$ analytic on the mentioned intervals.

ii). On the interval $\left(\tau^{\prime}, \tau^{\prime \prime}\right)$ there exist analytic bases $\left\{\mu \mapsto \mathcal{Y}_{j}^{ \pm}(\cdot, \mu)\right\}$ in the spaces of continuous spectrum eigenfunctions of problem (2.17) satisfying (3.8) and (3.9) with the scattering matrix $\mu \mapsto \mathcal{S}(\mu)$ analytic on $\left(\tau^{\prime}, \tau^{\prime \prime}\right)$.

Proof. In view of the argument in 3.2, it suffices to verify the analyticity of the scattering matrices. For example, let us consider the matrix $\mu \mapsto \mathcal{S}(\mu)$. Equality (3.19), the representation $w=v+c_{1} w_{1}^{-}+\cdots+c_{M} w_{M}^{-}$, and inclusion (3.18) lead to

$$
\mathcal{Y}_{j}^{+}(\cdot, \mu)=w_{j}^{+}(\cdot, \mu)-\sum_{k=1}^{M} c_{k}(\mu) w_{k}^{-}(\cdot, \mu) \in H_{\gamma}^{2}(G) .
$$


Therefore, $\mathcal{S}_{j k}(\mu)=-c_{k}(\mu), k=1, \ldots, M$. It remains to take into account that the functions $\mu \mapsto c_{k}(\mu)$ are analytic on $\left(\tau^{\prime}, \tau^{\prime \prime}\right)$.

For the basis $\left\{\mathcal{Y}_{j}^{+}(\cdot, \mu)\right\}_{j}^{M}$ (see Theorem 3.6, ii)), we introduce the columns $\mathcal{Y}_{(\mathbf{1})}^{+}=$ $\left(\mathcal{Y}_{1}^{+}, \ldots, \mathcal{Y}_{L}^{+}\right)^{t}$ and $\mathcal{Y}_{(\mathbf{2})}^{+}=\left(\mathcal{Y}_{L+1}^{+}, \ldots, \mathcal{Y}_{M}^{+}\right)^{t}$ and write down the scattering matrix in the form

$$
\mathcal{S}(\mu)=\left(\begin{array}{ll}
\mathcal{S}_{(11)}(\mu) & \mathcal{S}_{(12)}(\mu) \\
\mathcal{S}_{(21)}(\mu) & \mathcal{S}_{(22)}(\mu)
\end{array}\right)
$$

where $\mathcal{S}_{(11)}(\mu)$ is a block of size $L \times L$ and $\mathcal{S}_{(22)}(\mu)$ is a block of size $(M-L) \times(M-L)$, while $\mu \in\left(\tau^{\prime}, \tau^{\prime \prime}\right)$. We also set

$$
D=\left((\mu-\tau)^{1 / 2}+1\right) /\left((\mu-\tau)^{1 / 2}-1\right)
$$

with $(\mu-\tau)^{1 / 2}=i(\tau-\mu)^{1 / 2}$ for $\mu \leq \tau$ and $(\tau-\mu)^{1 / 2} \geq 0$. The next assertion will be of use in Section 4.

Lemma 3.7. Assume that $\mu \in\left(\tau^{\prime}, \tau\right]$ and $\mathcal{S}(\mu)$ is the scattering matrix in Theorem 3.6, ii). Then

$$
\begin{aligned}
\operatorname{ker}\left(D+\mathcal{S}_{(22)}(\mu)\right) & \subset \operatorname{ker} \mathcal{S}_{(12)}(\mu), \\
\operatorname{Im}\left(D+\mathcal{S}_{(22)}(\mu)\right) & \supset \operatorname{Im} \mathcal{S}_{(21)}(\mu) .
\end{aligned}
$$

Therefore the operator $\mathcal{S}_{(12)}(\mu)\left(D+\mathcal{S}_{(22)}(\mu)\right)^{-1}$ is defined on $\operatorname{Im}\left(D+\mathcal{S}_{(22)}(\mu)\right)$.

Proof. Let us consider (3.20). We assume that $h \in \operatorname{ker}\left(D+\mathcal{S}_{(22)}(\mu)\right)$ and $(0, h)^{t} \in \mathbb{C}^{M}$. Then

$$
\left(\begin{array}{ll}
\mathcal{S}_{(11)}(\mu) & \mathcal{S}_{(12)}(\mu) \\
\mathcal{S}_{(21)}(\mu) & \mathcal{S}_{(22)}(\mu)
\end{array}\right)\left(\begin{array}{l}
0 \\
h
\end{array}\right)=\left(\begin{array}{c}
S_{(12)}(\mu) h \\
-D h
\end{array}\right)
$$

Since the matrix $\mathcal{S}(\mu)$ is unitary and $|D|=1$, we have $\|h\|^{2}=\left\|\mathcal{S}_{(12)}(\mu) h\right\|^{2}+\|h\|^{2}$, so $\mathcal{S}_{(12)}(\mu) h=0$ and (3.20) is valid. Inclusion (3.21) is equivalent to

$$
\operatorname{ker}\left(D+\mathcal{S}_{(22)}(\mu)\right)^{*} \subset \operatorname{ker} \mathcal{S}_{(21)}(\mu)^{*} .
$$

Moreover,

$$
\mathcal{S}(\mu)^{*}=\left(\begin{array}{ll}
\mathcal{S}_{(11)}(\mu)^{*} & \mathcal{S}_{(21)}(\mu)^{*} \\
\mathcal{S}_{(12)}(\mu)^{*} & \mathcal{S}_{(22)}(\mu)^{*}
\end{array}\right)
$$

and the matrix $\mathcal{S}(\mu)^{*}$ is unitary, therefore (3.22) may be proven by the same argument as $(3.20)$.

\section{Other properties of the scattering matrices}

Here we clarify the connection between the matrices $\mathcal{S}(\mu)$ and $S(\mu)$ on the interval $\tau^{\prime}<\mu<$ $\tau$, prove the existence of the one-side finite limits $\lim S(\mu)$ as $\mu \rightarrow \tau \pm 0$, and describe the transformation of the scattering matrix under changes of basis in the space of waves $\mathcal{W}(\mu, G)$ for $\mu \in\left(\tau, \tau^{\prime \prime}\right)$. We keep the assumptions introduced at the very beginning of Section 3 . 


\subsection{The connection between $\mathcal{S}(\mu)$ and $S(\mu)$ for $\tau^{\prime}<\mu<\tau$}

Let us recall the description of the stable basis chosen for definition of $\mathcal{S}(\mu)$. In the semicylinder $\Pi_{+}^{1}$, we introduce the functions

$$
\Pi_{+}^{1} \ni(y, t) \mapsto e_{k}^{ \pm}(y, t ; \mu):=\chi(t) \exp \left( \pm i t \sqrt{\mu-\mu_{k}}\right) \varphi_{k}(y)
$$

where $k=l+1, \ldots, m$ (the notation is the same as in $(2.10)$; as before, $\mu_{l+1}=\cdots=\mu_{m}=\tau$ ). We extend the functions by zero to the whole domain $G$ and set

$$
w_{L+j}^{ \pm}(\cdot ; \mu)=2^{-1 / 2}\left(\frac{e_{l+j}^{+}(\cdot ; \mu)+e_{l+j}^{-}(\cdot ; \mu)}{2} \mp \frac{e_{l+j}^{+}(\cdot ; \mu)-e_{l+j}^{-}(\cdot ; \mu)}{2 \sqrt{\mu-\mu_{l+j}}}\right)
$$

for $j=1, \ldots, m-l=M-L$ (the equality $m-l=M-L$ was explained just after Remark 3.2). All the rest waves with supports in $\Pi_{+}^{1}$ that was obtained from the functions $(2.11)$ and the waves of the same type with supports in $\Pi_{+}^{2}, \ldots, \Pi_{+}^{\mathcal{T}}$, we number by one index $j=1, \ldots, L$ and denote by $w_{1}^{ \pm}(\cdot ; \mu), \ldots, w_{L}^{ \pm}(\cdot ; \mu)$. The obtained collection $\left\{w_{1}^{ \pm}, \ldots, w_{M}^{ \pm}\right\}$is a basis of waves in $G$ stable in a neighborhood of the threshold $\tau$. Finally, we introduce the columns $\mathbf{w}_{(\mathbf{1})}^{ \pm}=\left(w_{1}^{ \pm}, \ldots, w_{L}^{ \pm}\right)^{t}, \mathbf{w}_{(\mathbf{2})}^{ \pm}=\left(w_{L+1}^{ \pm}, \ldots, w_{M}^{ \pm}\right)^{t}$, and $\left(\mathbf{w}_{(\mathbf{1})}^{ \pm}, \mathbf{w}_{(\mathbf{2})}^{ \pm}\right)=\left(w_{1}^{ \pm}, \ldots, w_{M}^{ \pm}\right)^{t}$, where $t$ stands for matrix transposing. The components of the vector $\mathbf{w}_{(\mathbf{1})}^{ \pm}$are bounded, while the components of $\mathbf{w}_{(\mathbf{2})}^{ \pm}$exponentially grow at infinity in $\Pi_{+}^{1}$. Setting $\mathbf{e}_{(\mathbf{1})}^{ \pm}=\left(e_{1}^{ \pm}, \ldots, e_{L}^{ \pm}\right)^{t}$ and $\mathbf{e}_{(\mathbf{2})}^{ \pm}=\left(e_{L+1}^{ \pm}, \ldots, e_{M}^{ \pm}\right)^{t}$, we arrive at

$$
\mathbf{w}_{(\mathbf{2})}^{ \pm}=D^{\mp} \mathbf{e}_{(\mathbf{2})}^{+}+D^{ \pm} \mathbf{e}_{(\mathbf{2})}^{-}
$$

with

$$
D^{ \pm}=\left((\mu-\tau)^{1 / 2} \pm 1\right) / 2 \sqrt{2}(\mu-\tau)^{1 / 2} .
$$

The following assertion is, in essence, contained in [7].

Proposition 4.1. Let $\mu \in\left(\tau^{\prime}, \tau\right)$ and let $S(\mu)$ and $\mathcal{S}(\mu)$ be the scattering matrices in Theorem 3.6. Then

$$
S(\mu)=\mathcal{S}_{(11)}(\mu)-\mathcal{S}_{(12)}(\mu)\left(D+\mathcal{S}_{(22)}(\mu)\right)^{-1} \mathcal{S}_{(21)}(\mu),
$$

with

$$
D=D^{+} / D^{-}=\left((\mu-\tau)^{1 / 2}+1\right) /\left((\mu-\tau)^{1 / 2}-1\right) .
$$

Proof. We verify (4.4). Rewrite (3.8) in the form

$$
\begin{gathered}
\mathcal{Y}_{(\mathbf{1})}^{+}-\mathbf{w}_{(\mathbf{1})}^{+}-\mathcal{S}_{(11)} \mathbf{w}_{(\mathbf{1})}^{-}-\mathcal{S}_{(12)} \mathbf{w}_{(\mathbf{2})}^{-} \in H_{\gamma}^{2}(G), \\
\mathcal{Y}_{(\mathbf{2})}^{+}-\mathbf{w}_{(\mathbf{2})}^{+}-\mathcal{S}_{(21)} \mathbf{w}_{(\mathbf{1})}^{-}-\mathcal{S}_{(22)} \mathbf{w}_{(\mathbf{2})}^{-} \in H_{\gamma}^{2}(G) .
\end{gathered}
$$

Recall that $\gamma>0$ has been chosen according to Lemma 3.4, so the strip $\{\lambda \in \mathbb{C}:|\operatorname{Im} \lambda|<\gamma\}$ contains the eigenvalues $\pm(\mu-\tau)^{1 / 2}$ of the pencil $\mathfrak{A}^{1}(\cdot, \mu)$. We take $\delta>0$ such that the strip $\{\lambda \in \mathbb{C}:|\operatorname{Im} \lambda|<\delta\}$ contains only the real eigenvalues of the pencils $\mathfrak{A}^{r}(\cdot, \mu), r=1, \ldots, \mathcal{T}$; then $\delta<\gamma$ and $H_{\gamma}^{2}(G) \subset H_{\delta}^{2}(G)$. Instead of $\mathbf{w}_{(\mathbf{2})}^{ \pm}$, we substitute into (4.5) their expressions 
in (4.3); for the aforementioned $\delta$ the vector-valued function $\mathbf{e}_{(\mathbf{2})}^{+}$belongs to $H_{\delta}^{2}(G)$. As a result we obtain

$$
\begin{gathered}
\mathcal{Y}_{(\mathbf{1})}^{+}=\mathbf{w}_{(\mathbf{1})}^{+}+\mathcal{S}_{(11)} \mathbf{w}_{(\mathbf{1})}^{-}+\mathcal{S}_{(12)} D^{-} \mathbf{e}_{(\mathbf{2})}^{-}+\Re_{(\mathbf{1})}, \\
\mathcal{Y}_{(\mathbf{2})}^{+}=\mathcal{S}_{(21)} \mathbf{w}_{(\mathbf{1})}^{-}+\left(D+\mathcal{S}_{(22)}\right) D^{-} \mathbf{e}_{(\mathbf{2})}^{-}+\Re_{(\mathbf{2})},
\end{gathered}
$$

where $\Re_{(\mathbf{1})}, \Re_{(\mathbf{2})} \in H_{\delta}^{2}(G)$. Introduce the orthogonal projector

$$
\mathcal{P}: \mathbb{C}^{M-L} \rightarrow \operatorname{Im}\left(D+\mathcal{S}_{(22)}(\mu)\right) .
$$

Taking account of (3.21) and (4.7), we arrive at

$$
\mathcal{P} \mathcal{Y}_{(\mathbf{2})}^{+}=\mathcal{S}_{(21)} \mathbf{w}_{(\mathbf{1})}^{-}+\left(D+\mathcal{S}_{(22)}\right) D^{-} \mathbf{e}_{(\mathbf{2})}^{-}+\mathcal{P} \Re_{(\mathbf{2})}
$$

We apply the operator $\mathcal{S}_{(12)}(\mu)\left(D+\mathcal{S}_{(22)}(\mu)\right)^{-1}$ to both sides of (4.8) and subtract the resulting equality from (4.6). We have

$$
Z=\mathbf{w}_{(\mathbf{1})}^{+}+\left(\mathcal{S}_{(11)}(\mu)-\mathcal{S}_{(12)}(\mu)\left(D+\mathcal{S}_{(22)}(\mu)\right)^{-1} \mathcal{S}_{(21)}(\mu)\right) \mathbf{w}_{(\mathbf{1})}^{-}+R
$$

where

$$
\begin{aligned}
& Z=\mathcal{Y}_{(\mathbf{1})}^{+}-\mathcal{S}_{(12)}(\mu)\left(D+\mathcal{S}_{(22)}(\mu)\right)^{-1} \mathcal{P} \mathcal{Y}_{(\mathbf{2})}^{+}, \\
& R=\Re_{(\mathbf{1})}-\mathcal{S}_{(12)}(\mu)\left(D+\mathcal{S}_{(22)}(\mu)\right)^{-1} \mathcal{P} \Re_{(\mathbf{2})} .
\end{aligned}
$$

The components of the vectors $\mathcal{Y}_{(\mathbf{1})}^{+}$and $\mathcal{Y}_{(\mathbf{2})}^{+}$satisfy problem (2.17); in view of (4.10), the same is true for the components of the vector $Z$. Moreover from $\Re_{(\mathbf{1})}, \Re_{(\mathbf{2})} \in H_{\delta}^{2}(G)$, and (4.11) it follows that $R \in H_{\delta}^{2}(G)$. Hence the formula (4.9) describes the scattering of the vector $\mathbf{w}_{(\mathbf{1})}^{+}$of incoming waves in the basis $\mathbf{w}_{(\mathbf{1})}^{+}, \mathbf{w}_{(\mathbf{1})}^{-}$as well as (3.3), so we obtain (4.4).

\subsection{The connection between $\mathcal{S}(\mu)$ and $S(\mu)$ for $\tau<\mu<\tau^{\prime \prime}$}

We consider two bases in the wave space $\mathcal{W}(\mu, G)$ for $\tau<\mu<\tau^{\prime \prime}$. One of the bases consists of the waves in $G$ corresponding to functions of the form $u_{q}^{ \pm}(\cdot, \mu)$ in $(2.6)$, while the other one comprises the waves generated by the functions $w_{q}^{ \pm}(\cdot, \mu)$ (see $(2.10),(2.11)$ ). As before, the scattering matrices defined in these bases are denoted by $S(\mu)$ and $\mathcal{S}(\mu)$ (see Theorem $3.6)$; this time, that is, for $\mu \in\left(\tau, \tau^{\prime \prime}\right)$, the matrices are of the same size $M \times M$.

The scattering matrices are independent of the choice of the cut-off function $\chi$ in the definition of the space $\mathcal{W}(\mu, G)$. Identifying "equivalent" waves, one can omit such a cut-off function from consideration. To this end we introduce the quotient space

$$
\dot{\mathcal{W}}(\mu, G):=\left(\mathcal{W}(\mu, G) \dot{+} H_{\gamma}^{2}(G)\right) / H_{\gamma}^{2}(G)
$$

Let $\dot{v}$ stand for the class in $\dot{\mathcal{W}}(\mu, G)$ with representative $v \in \mathcal{W}(\mu, G)$. In what follows, waves of the form $\chi u_{q}^{ \pm}(\cdot, \mu)$ and $\chi w_{q}^{ \pm}(\cdot, \mu)$ in $G$ are denoted by $u_{q}^{ \pm}(\cdot, \mu)$ and $w_{q}^{ \pm}(\cdot, \mu)$. The collections $\left\{\dot{u}_{q}^{ \pm}(\cdot, \mu)\right\}_{j=1}^{M}$ and $\left\{\dot{w}_{k}^{ \pm}(\cdot, \mu)\right\}_{k=1}^{M}$ are bases in the space $\dot{\mathcal{W}}(\mu, G)$, so $\operatorname{dim} \dot{\mathcal{W}}(\mu, G)=2 M$. The 
form $q_{G}(u, v)$ in (2.18) is independent of the choice of representatives in $\dot{u}$ and $\dot{v}$; hence it is defined on $\dot{\mathcal{W}}(\mu, G) \times \dot{\mathcal{W}}(\mu, G)$. From (2.8) and (2.9) it follows that

$$
\begin{aligned}
& i q_{G}\left(\dot{u}_{k}^{ \pm}(\cdot ; \mu), \dot{u}_{l}^{\mp}(\cdot ; \mu)\right)=0 \quad \text { for all } k, l=1, \ldots, M, \\
& i q_{G}\left(\dot{u}_{k}^{ \pm}(\cdot ; \mu), \dot{u}_{l}^{ \pm}(\cdot ; \mu)\right)=\mp \delta_{k l},
\end{aligned}
$$

while (2.12) and (2.13) lead to

$$
\begin{aligned}
& i q_{G}\left(\dot{w}_{r}^{ \pm}(\cdot ; \mu), \dot{w}_{s}^{\mp}(\cdot ; \mu)\right)=0 \text { for all } r, s=1, \ldots, M, \\
& i q_{G}\left(\dot{w}_{r}^{ \pm}(\cdot ; \mu), \dot{w}_{s}^{ \pm}(\cdot ; \mu)\right)=\mp \delta_{r s} .
\end{aligned}
$$

Thus $\dot{\mathcal{W}}(\mu, G)$ turns out to be a $2 M$-dimensional complex space with indefinite inner product $<\dot{u}, \dot{v}>:=-i q_{G}(\dot{u}, \dot{v})$. The projection

$$
\pi: \mathcal{W}(\mu, G) \dot{+} H_{\gamma}^{2}(G) \rightarrow \dot{\mathcal{W}}(\mu, G)
$$

maps the space of continuous spectrum eigenfunctions onto a subspace in $\dot{\mathcal{W}}(\mu, G)$ of dimension $M$; denote the subspace by $\mathcal{E}(\mu)$.

Let $V_{1}, \ldots, V_{2 M}$ be a basis in $\dot{\mathcal{W}}(\mu, G)$ subject to the orthogonality and normalization conditions

$$
<V_{j}, V_{l}>=\delta_{j l},<V_{j+M}, V_{l+M}>=-\delta_{j l} \text { for } j, l=1, \ldots, M \text {. }
$$

The elements $V_{1}, \ldots, V_{M}$ are called incoming waves while the elements $V_{M+1}, \ldots, V_{2 M}$ are called outgoing waves. Assume that $X_{1}, \ldots, X_{M}$ is a basis of $\mathcal{E}(\mu)$ that defines, in the basis of waves $V_{1}, \ldots, V_{2 M}$, the scattering matrix $\mathfrak{S}(\mu)$ of size $M \times M$ (compare with (3.3)). We represent the vectors $X_{j}$ as coordinate rows and form the $M \times 2 M$-matrix $X=\left(X_{1}, \ldots, X_{M}\right)^{t}$ (which is a column of the letters $X_{1}, \ldots, X_{M}$ ). Finally, let $I$ stand for the unit matrix of size $M \times M$. Then a relation of the form (3.3) leads to

$$
X=(I, \mathfrak{S}(\mu)) V
$$

where $V$ is the $2 M \times 2 M$-matrix $\left(V_{1}, \ldots, V_{2 M}\right)^{t}$ consisting of the coordinate rows of the vectors $V_{j}$ and $(I, \mathfrak{S}(\mu))$ is a matrix of size $M \times 2 M$.

Assume that $\tilde{V}_{1}, \ldots, \tilde{V}_{2 M}$ is another basis of waves subject to conditions of the form (4.17), $\tilde{X}_{1}, \ldots, \tilde{X}_{M}$ is a basis of $\mathcal{E}(\mu)$, and $\tilde{\mathfrak{S}}(\mu)$ is the corresponding scattering matrix such that

$$
\tilde{X}=(I, \tilde{\mathfrak{S}}(\mu)) \tilde{V}
$$

We suppose that $\tilde{V}=\mathfrak{T} V$ and write down the $2 M \times 2 M$-matrix $\mathfrak{T}$ as $\mathfrak{T}=\left(\mathfrak{T}_{(k l)}\right)_{k, l=1}^{2}$ with blocks $\mathfrak{T}_{(k l)}$ of size $M \times M$.

Lemma 4.2. The matrices $\mathfrak{T}_{(11)}+\tilde{\mathfrak{S}}(\mu) \mathfrak{T}_{(21)}$ and $\mathfrak{T}_{(12)}+\tilde{\mathfrak{S}}(\mu) \mathfrak{T}_{(22)}$ are invertible and

$$
\mathfrak{S}(\mu)=\left(\mathfrak{T}_{(11)}+\tilde{\mathfrak{S}}(\mu) \mathfrak{T}_{(21)}\right)^{-1}\left(\mathfrak{T}_{(12)}+\tilde{\mathfrak{S}}(\mu) \mathfrak{T}_{(22)}\right) .
$$


Proof. For the bases $X_{1}, \ldots, X_{M}$ and $\tilde{X}_{1}, \ldots, \tilde{X}_{M}$ there exists a nonsingular $M \times M$-matrix $B$ such that $\tilde{X}=B X$. Therefore, by virtue of (4.19), we have

$$
B X=(I, \tilde{\mathfrak{S}}(\mu)) \mathfrak{T} V .
$$

Taking account of (4.18), we obtain $B(I, \mathfrak{S}(\mu)) V=(I, \tilde{\mathfrak{S}}(\mu)) \mathfrak{T} V$, so

$$
B(I, \mathfrak{S}(\mu))=(I, \tilde{\mathfrak{S}}(\mu)) \mathfrak{T} .
$$

Let us write this equality in the form

$$
(B, B \mathfrak{S}(\mu))=\left(\mathfrak{T}_{(11)}+\tilde{\mathfrak{S}}(\mu) \mathfrak{T}_{(21)}, \mathfrak{T}_{(12)}+\tilde{\mathfrak{S}}(\mu) \mathfrak{T}_{(22)}\right)
$$

Now the assertions of lemma are evident.

We intend to make use of (4.20) taking as $\tilde{V}$ the image, under canonical projection (4.16), of the stable basis of $\mathcal{W}(\mu, G)$ in (3.8) and as $V$ the image of the wave basis in (3.3). As $\tilde{\mathfrak{S}}(\mu)$ and $\mathfrak{S}(\mu)$, we choose $\mathcal{S}(\mu)$ and $S(\mu)$ respectively. We proceed to computing the matrix $\mathfrak{T}$ in the equality $\tilde{V}=\mathfrak{T} V$. In doing so, instead of $\tilde{V}$ and $V$ we can consider their just mentioned preimages in $\mathcal{W}(\mu, G)$. We set

$$
u_{j}:=u_{j}^{+}, \quad u_{j+M}:=u_{j}^{-}, \quad j=1, \ldots, M,
$$

where $u_{j}^{ \pm}$are the waves in $\mathcal{W}(\mu, G)$ generated by functions of the form (2.6). We also introduce

$$
\begin{array}{r}
w_{j}:=w_{j}^{+}=u_{j}^{+}, \quad w_{j+M}:=w_{j}^{-}=u_{j}^{-}, \quad j=1, \ldots, L, \\
w_{p}:=w_{p}^{+}, \quad w_{p+M}:=w_{p}^{-}, \quad p=L+1, \ldots, M,
\end{array}
$$

where $w_{p}^{ \pm}$are the waves in $\mathcal{W}(\mu, G)$ generated by functions $(2.10)$. For the matrix $\mathfrak{T}$, the equality $w=\mathfrak{T} u$ holds with the columns $w=\left(w_{1}, \ldots, w_{2 M}\right)^{t}$ and $u=\left(u_{1}, \ldots, u_{2 M}\right)^{t}$. For convenience, we will here denote functions (2.10) in the same way as the waves $w_{p}^{ \pm}$; let us write down functions in the form

$$
\left.w_{p}^{ \pm}(\mu)=2^{-1 / 2}\left(\left(e^{i t \lambda}+e^{-i t \lambda}\right) / 2\right) \mp\left(e^{i t \lambda}-e^{-i t \lambda}\right) / 2 \lambda\right) \varphi_{p}
$$

where $\lambda=\sqrt{\mu-\tau}$ and $\tau$ is a threshold; we also write the functions (2.6) in the form

$$
u_{p}^{ \pm}(\mu)=(2 \lambda)^{-1 / 2} e^{\mp i t \lambda} \varphi_{p}
$$

Then we have

$$
w_{p}^{ \pm}=(1 / 2)\left(u_{p}^{+}\left(\lambda^{1 / 2} \pm \lambda^{-1 / 2}\right)+u_{p}^{-}\left(\lambda^{1 / 2} \mp \lambda^{-1 / 2}\right)\right), \quad p=L+1, \ldots, M
$$

here by $w_{p}^{ \pm}$and $u_{p}^{ \pm}$one can mean the functions in the cylinder and the corresponding waves in the domain $G$ alike. Together with (4.21) and (4.22), this leads to the following description of the blocks $\mathfrak{T}_{i j}$ of the matrix $\mathfrak{T}$. 
Lemma 4.3. Each of the matrices $\mathfrak{T}_{(i j)}$ consists of four blocks and is block-diagonal. The equalities

$$
\begin{array}{r}
\mathfrak{T}_{(11)}(\mu)=\mathfrak{T}_{(22)}(\mu)=\operatorname{diag}\left\{I_{L}, 2^{-1}\left(\lambda^{(1 / 2)}+\lambda^{(-1 / 2)}\right) I_{M-L}\right\} \\
\mathfrak{T}_{(21)}(\mu)=\mathfrak{T}_{(12)}(\mu)=\operatorname{diag}\left\{O_{L}, 2^{-1}\left(\lambda^{1 / 2}-\lambda^{-1 / 2}\right) I_{M-L}\right\}
\end{array}
$$

hold, where $I_{K}$ is the unit matrix of size $K \times K, O_{L}$ is the zero matrix of size $L \times L$, and $\lambda=\sqrt{\mu-\tau}$ with $\mu \in\left(\tau, \tau^{\prime \prime}\right)$.

We return to $(4.20)$ with $\mathcal{S}$ and $S$ instead of $\tilde{\mathfrak{S}}$ and $\mathfrak{S}$. Let us divide the matrix $\mathcal{S}$ into four blocks with $\mathcal{S}_{(11)}$ of size $L \times L$ and $\mathcal{S}_{(22)}$ of size $(M-L) \times(M-L)$. We also set $d^{ \pm}=2^{-1}\left(\lambda^{1 / 2} \pm \lambda^{-1 / 2}\right)$. Then

$$
\mathfrak{T}_{(11)}+\mathcal{S} \mathfrak{T}_{(21)}=\left(\begin{array}{cc}
I_{L} & \mathcal{S}_{(12)} d^{-} \\
O & \mathcal{S}_{(22)} d^{-}+I_{M-L} d^{+}
\end{array}\right)
$$

According to Lemma 4.2 , the matrix $\mathfrak{T}_{(11)}+\mathcal{S} \mathfrak{T}_{(21)}$ is invertible, so the matrix $\mathcal{S}_{(22)} d^{-}+$ $I_{M-L} d^{+}$is invertible as well, therefore

$$
\left(\mathfrak{T}_{(11)}+\mathcal{S} \mathfrak{T}_{(21)}\right)^{-1}=\left(\begin{array}{cc}
I_{L} & -\mathcal{S}_{(12)} d^{-}\left(\mathcal{S}_{(22)} d^{-}+I_{M-L} d^{+}\right)^{-1} \\
O & \left(\mathcal{S}_{(22)} d^{-}+I_{M-L} d^{+}\right)^{-1}
\end{array}\right)
$$

In view of (4.20) we now obtain

Proposition 4.4. For $\mu \in\left(\tau, \tau^{\prime \prime}\right)$ the blocks $S_{(i j)}$ of the scattering matrix

$$
S(\mu)=\left(\mathfrak{T}_{(11)}+\mathcal{S}(\mu) \mathfrak{T}_{(21)}\right)^{-1}\left(\mathfrak{T}_{(12)}+\mathcal{S}(\mu) \mathfrak{T}_{(22)}\right)
$$

admit the representations

$$
\begin{aligned}
S_{(11)} & =\mathcal{S}_{(11)}-\mathcal{S}_{(12)} d^{-}\left(\mathcal{S}_{(22)} d^{-}+I_{M-L} d^{+}\right)^{-1} \mathcal{S}_{(21)} \\
S_{(12)} & =\mathcal{S}_{(12)} d^{+}-\mathcal{S}_{(12)} d^{-}\left(\mathcal{S}_{(22)} d^{-}+I_{M-L} d^{+}\right)^{-1}\left(\mathcal{S}_{(22)} d^{+}+I_{M-L} d^{-}\right) \\
S_{(21)} & =\left(\mathcal{S}_{(22)} d^{-}+I_{M-L} d^{+}\right)^{-1} \mathcal{S}_{(21)} \\
S_{(22)} & =\left(\mathcal{S}_{(22)} d^{-}+I_{M-L} d^{+}\right)^{-1}\left(\mathcal{S}_{(22)} d^{+}+I_{M-L} d^{-}\right)
\end{aligned}
$$

\subsection{The limits of $S(\mu)$ as $\mu \rightarrow \tau \pm 0$}

To calculate the one-sided limits of $S(\mu)$, we make use of (4.4) as $\mu \rightarrow \tau-0$ and apply (4.27) - (4.30) as $\mu \rightarrow \tau+0$. The computation procedure depends on whether the number 1 is an eigenvalue of the matrix $\mathcal{S}_{22}(\tau)$.

\subsubsection{The limits of $S(\mu)$ as $\mu \rightarrow \tau \pm 0$ provided 1 is not an eigenvalue of $\mathcal{S}_{(22)}(\tau)$}

Recall that the functions $\mu \mapsto \mathcal{S}_{(k l)}(\mu)$ are analytic in a neighborhood of $\mu=\tau$. Therefore from (4.4) it immediately follows that

$$
\lim _{\mu \rightarrow \tau-0} S(\mu)=\mathcal{S}_{(11)}(\tau)-\mathcal{S}_{(12)}(\tau)\left(\mathcal{S}_{(22)}(\tau)-1\right)^{-1} \mathcal{S}_{(21)}(\tau)
$$


Let us proceed to compute $\lim S(\mu)$ as $\mu \rightarrow \tau+0$. By virtue of (4.27) and (4.31),

$$
\begin{array}{r}
\lim _{\mu \rightarrow \tau+0} S_{(11)}(\mu)=\lim _{\mu \rightarrow \tau+0}\left(\mathcal{S}_{(11)}(\mu)-\mathcal{S}_{(12)}(\mu)\left(\mathcal{S}_{(22)}(\mu)+d^{+}(\mu) / d^{-}(\mu)\right)^{-1} \mathcal{S}_{(21)}(\mu)\right) \\
=\mathcal{S}_{(11)}(\tau)-\mathcal{S}_{(12)}(\tau)\left(\mathcal{S}_{(22)}(\tau)-1\right)^{-1} \mathcal{S}_{(21)}(\tau)=\lim _{\mu \rightarrow \tau-0} S(\mu)
\end{array}
$$

According to (4.30),

$$
\begin{aligned}
\lim _{\mu \rightarrow \tau+0} S_{(22)}(\mu) & =\lim _{\mu \rightarrow \tau+0}\left(\mathcal{S}_{(22)}+d^{+} / d^{-}\right)^{-1}\left(\mathcal{S}_{(22)} d^{+} / d^{-}+1\right) \\
= & \left(\mathcal{S}_{(22)}(\tau)-1\right)^{-1}\left(-\mathcal{S}_{(22)}(\tau)+1\right)=-I_{M-L}
\end{aligned}
$$

It follows from (4.29) that

$$
S_{(21)}(\mu)=\left(\mathcal{S}_{(22)}+d^{+} / d^{-}\right)^{-1} \mathcal{S}_{(21)} / d^{-} .
$$

Since $d^{-}(\mu)=2^{-1}\left((\mu-\tau)^{1 / 2}-1\right) /(\mu-\tau)^{1 / 4}$, we arrive at

$$
S_{(21)}(\mu)=O\left((\mu-\tau)^{1 / 4}\right) \rightarrow 0 \quad \text { for } \quad \mu \rightarrow \tau+0 .
$$

Finally, consider $S_{(12)}(\mu)$. We rewrite $(4.28)$ in the form

$S_{(12)}=\mathcal{S}_{(12)} d^{+}\left(1-\left(\mathcal{S}_{(22)}+d^{+} / d^{-}\right)^{-1}\left(\mathcal{S}_{(22)}+d^{-} / d^{+}\right)\right)=\mathcal{S}_{(12)} d^{+}\left(\mathcal{S}_{(22)}+d^{+} / d^{-}\right)^{-1}\left(d^{+} / d^{-}-d^{-} / d^{+}\right)$.

In view of

$$
d^{+}(\mu)\left(d^{+} / d^{-}-d^{-} / d^{+}\right)=2(\mu-\tau)^{1 / 4} /\left((\mu-\tau)^{1 / 2}-1\right),
$$

we obtain

$$
S_{(12)}(\mu)=O\left((\mu-\tau)^{1 / 4}\right) \rightarrow 0 \quad \text { for } \quad \mu \rightarrow \tau+0 .
$$

\subsubsection{The limits of $S(\mu)$ as $\mu \rightarrow \tau \pm 0$ provided 1 is an eigenvaue of $\mathcal{S}_{(22)}(\tau)$}

We set $\lambda=\sqrt{\mu-\tau}$ with $\mu=\tau+\lambda^{2}$ and consider the function $\lambda \mapsto \Phi(\lambda): \mathbb{C}^{M-L} \rightarrow \mathbb{C}^{M-L}$,

$$
\Phi(\lambda):=\mathcal{S}_{(22)}(\mu)+d^{+}(\mu) / d^{-}(\mu)=\mathcal{S}_{(22)}\left(\tau+\lambda^{2}\right)+(\lambda+1) /(\lambda-1) .
$$

The number $\lambda=0$ is an eigenvalue of the function $\lambda \mapsto \Phi(\lambda)$, if and only if 1 is an eigenvalue of the matrix $\mathcal{S}_{(22)}(\tau)$; in such a case $\operatorname{ker}\left(\mathcal{S}_{(22)}(\tau)-1\right)=\operatorname{ker} \Phi(0)$. To calculate the limits of $S(\mu)$ as $\mu \rightarrow \tau \pm 0$, we need a knowledge of the resolvent $\lambda \mapsto \Phi(\lambda)^{-1}$ in a neighborhood of $\lambda=0$. Propositions 4.5 and 4.6 provide the required information.

Proposition 4.5. There holds the equality

$$
\operatorname{ker}\left(\mathcal{S}_{(22)}(\tau)-1\right)=\operatorname{ker}\left(\mathcal{S}_{(22)}(\tau)^{*}-1\right) .
$$

Proof. Assume that $h \in \operatorname{ker}\left(\mathcal{S}_{(22)}(\tau)-1\right)$. Then, as was shown in the proof of Lemma 3.7, the vector $(0, h)^{t} \in \mathbb{C}^{M}$ belongs to $\operatorname{ker}(\mathcal{S}(\tau)-1)$ and $\mathcal{S}_{(12)}(\tau) h=0$. The same argument with $\mathcal{S}(\tau)^{*}$ instead of $\mathcal{S}(\tau)$ shows that the inclusion $g \in \operatorname{ker}\left(\mathcal{S}_{(22)}(\tau)^{*}-1\right)$ implies $(0, g)^{t} \in$ $\operatorname{ker}\left(\mathcal{S}(\tau)^{*}-1\right)$ and $\mathcal{S}_{(21)}(\tau)^{*} g=0$. Since $\mathcal{S}(\tau)^{*}=\mathcal{S}(\tau)^{-1}$, we have

$$
\operatorname{ker}(\mathcal{S}(\tau)-1)=\operatorname{ker}\left(\mathcal{S}(\tau)^{*}-1\right) .
$$


Let $h_{1}, \ldots, h_{\varkappa}$ be a basis of $\operatorname{ker}\left(\mathcal{S}_{(22)}(\tau)-1\right)$ and $g_{1}, \ldots, g_{\varkappa}$ the basis of $\operatorname{ker}\left(\mathcal{S}_{(22)}(\tau)^{*}-1\right)$. We set $\tilde{h_{j}}=\left(0, h_{j}\right)^{t}$ and $\tilde{g}_{j}=\left(0, g_{j}\right)^{t}$. From (4.38) it follows that

$$
\tilde{h_{j}}, \tilde{g}_{j} \in \operatorname{ker}(\mathcal{S}(\tau)-1)=\operatorname{ker}\left(\mathcal{S}(\tau)^{*}-1\right), \quad j=1, \ldots, \varkappa .
$$

Therefore, any vector of the collection $h_{1}, \ldots, h_{\varkappa}$ is a linear combination of the vectors $g_{1}, \ldots, g_{\varkappa}$ and vice versa.

Proposition 4.6. Let $\Phi$ be the matrix function in (4.36) and $\operatorname{dim} \operatorname{ker} \Phi(0)=\varkappa>0$. Then, in a punctured neighborhood of $\lambda=0$, the resolvent $\lambda \mapsto \Phi(\lambda)^{-1}$ admits the representation

$$
\Phi(\lambda)^{-1}=-(2 \lambda)^{-1} \sum_{j=1}^{\varkappa}\left\{\cdot, h_{j}\right\} h_{j}+\Gamma(\lambda)
$$

here $h_{1}, \ldots, h_{\varkappa}$ is an orthonormal basis of $\operatorname{ker}\left(\mathcal{S}_{(22)}(\tau)-1\right),\{u, v\}$ is the inner product on the space $\mathbb{C}^{M-L}$, and $\lambda \mapsto \Gamma(\lambda): \mathbb{C}^{M-L} \rightarrow \mathbb{C}^{M-L}$ is a matrix function holomorphic in $a$ neighborhood of $\lambda=0$.

Proof. It is known (e.g., see [9],[10]) that, under certain conditions, the resolvent $\mathfrak{A}(\lambda)^{-1}$ of a holomorphic operator function $\lambda \mapsto \mathfrak{A}(\lambda)$ in a punctured neighborhood of an isolated eigenvalue $\lambda_{0}$ admits the representation

$$
\mathfrak{A}(\lambda)^{-1}=\left(\lambda-\lambda_{0}\right)^{-1} \sum_{j=1}^{\varkappa}\left(\cdot, \psi_{j}\right) \phi_{j}+\Gamma(\lambda),
$$

where $\phi_{1}, \ldots, \phi_{\varkappa}$ and $\psi_{1}, \ldots, \psi_{\varkappa}$ are bases of the spaces $\operatorname{ker} \mathfrak{A}\left(\lambda_{0}\right)$ and $\operatorname{ker} \mathfrak{A}\left(\lambda_{0}\right)^{*}$ satisfying the orthogonality and normalization conditions

$$
\left(\partial_{\lambda} \mathfrak{A}\left(\lambda_{0}\right) \phi_{j}, \psi_{k}\right)=\delta_{j k}, \quad j, k=1, \ldots, \varkappa
$$

and $\Gamma$ is an operator function holomorphic in a neighborhood of $\lambda_{0}$. Formula (4.40) is related to the case where the operator function $\lambda \mapsto \mathfrak{A}(\lambda)$ has no generalized eigenvectors at the point $\lambda_{0}$. To justify (4.39), we have to show that there are no generalized eigenvectors of the function $\lambda \mapsto \Phi(\lambda)$ at the point $\lambda=0$ and to verify agreement between (4.39) and (4.40).

We first take up the generalized eigenvectors. Assume that $0 \neq h^{0} \in \operatorname{ker} \Phi(0)$. The equation $\Phi(0) h^{1}+\left(\partial_{\lambda} \Phi\right)(0) h^{0}=0$ for a generalized eigenvector $h^{1}$ is of the form

$$
\left(\mathcal{S}_{(22)}(\tau)-1\right) h^{1}=2 h^{0} .
$$

The orthogonality of $h^{0}$ to the lineal $\operatorname{ker}\left(\mathcal{S}_{(22)}(\tau)^{*}-1\right)=\operatorname{ker}\left(\mathcal{S}_{(22)}(\tau)-1\right)$ is necessary for the solvability of this equation (see (4.37)). Since $0 \neq h^{0} \in \operatorname{ker} \Phi(0)=\operatorname{ker}\left(\mathcal{S}_{(22)}(\tau)-1\right)$, the solvability condition is not fulfilled, so the generalized eigenvectors do not exist.

Let us compare (4.39) and (4.40). We have $\left(\partial_{\lambda} \Phi\right)(0)=-2 I_{M-L}$. Moreover, in view of $(4.37)$, the bases $\phi_{1}, \ldots, \phi_{\varkappa}$ and $\psi_{1}, \ldots, \psi_{\varkappa}$ in (4.40) can be chosen to satisfy $\phi_{j}=-\psi_{j}=$ $h_{j} / \sqrt{2}$ and as $h_{1}, \ldots, h_{\varkappa}$ there can be taken an orthonormal basis of $\operatorname{ker}\left(\mathcal{S}_{22}(\tau)-1\right)$. Then

$$
\left\{\left(\partial_{\lambda} \Phi\right)(0) \phi_{j}, \psi_{k}\right\}=\delta_{j k}, \quad j, k=1, \ldots, \varkappa,
$$


and the representation (4.40) takes the form of (4.39).

Let us calculate $\lim S(\mu)$ as $\mu \rightarrow \tau-0$. According to Lemma 3.7,

$$
\operatorname{Im}\left(\mathcal{S}_{(22)}(\tau)-1\right) \supset \operatorname{Im} \mathcal{S}_{(21)}(\tau) .
$$

Therefore, Proposition 4.5 leads to the equalities $\left\{\mathcal{S}_{(21)}(\tau) f, h_{j}\right\}=0$ for any $f \in \mathbb{C}^{L}$ and $h_{1}, \ldots, h_{\varkappa}$ in (4.39). Because the function $\mu \rightarrow \mathcal{S}_{(21)}(\mu)$ is analytic, we have $\mathcal{S}_{(21)}(\mu)=$ $\mathcal{S}_{(21)}(\tau)+O(|\mu-\tau|)$; recall that $|\mu-\tau|=|\lambda|^{2}$. Applying (4.39), we obtain

$$
\left(\mathcal{S}_{(22)}(\mu)+D(\mu)\right)^{-1} \mathcal{S}_{(21)}(\mu)=\Gamma(\lambda) \mathcal{S}_{(21)}(\mu)+O(|\lambda|) .
$$

Now from (4.4) it follows that

$$
\lim _{\mu \rightarrow \tau-0} S(\mu)=\mathcal{S}_{(11)}(\tau)-\mathcal{S}_{(12)}(\tau) \Gamma(0) \mathcal{S}_{(21)}(\tau)
$$

Lemma 3.7 allows to treat the right-hand side as the operator $\mathcal{S}_{(11)}(\tau)-\mathcal{S}_{(12)}(\tau)\left(\mathcal{S}_{(22)}(\tau)-\right.$ $1)^{-1} \mathcal{S}_{(21)}(\tau)$ (see $\left.(4.31)\right)$. For $\mu \rightarrow \tau-0$ there holds the estimate

$$
S(\mu)-\left(\mathcal{S}_{(11)}(\tau)-\mathcal{S}_{(12)}(\tau) \Gamma(0) \mathcal{S}_{(21)}(\tau)\right)=O\left(|\mu-\tau|^{(1 / 2)}\right)
$$

Let us proceed to calculating the limits as $\mu \rightarrow \tau+0$. We compute $\lim _{\mu \rightarrow \tau+0} S_{(11)}(\mu)$ in the same way as $\lim _{\mu \rightarrow \tau-0} S(\mu)$ and obtain

$$
\lim _{\mu \rightarrow \tau+0} S_{(11)}(\mu)=\lim _{\mu \rightarrow \tau-0} S(\mu)
$$

In view of $(4.30)$,

$$
\begin{aligned}
& S_{(22)}(\mu)=\left(\mathcal{S}_{(22)}(\mu)+d^{+} / d^{-}\right)^{-1}\left(\mathcal{S}_{(22)}(\mu)+d^{-} / d^{+}\right) d^{+} / d^{-} \\
& =d^{+} / d^{-}+\left(\mathcal{S}_{(22)}(\mu)+d^{+} / d^{-}\right)^{-1}\left(d^{-} / d^{+}-d^{+} / d^{-}\right) d^{+} / d^{-}
\end{aligned}
$$

Applying resolvent representation (4.39), we write the last equality in the form

$$
S_{(22)}(\mu)=\frac{\lambda+1}{\lambda-1}\left(I+\frac{2}{\lambda^{2}-1} \sum_{j=1}^{\varkappa}\left(\cdot, h_{j}\right) h_{j}-\frac{4 \lambda}{\lambda^{2}-1} \Gamma(\lambda)\right) .
$$

Hence

$$
\lim _{\mu \rightarrow \tau+0} S_{(22)}(\mu)=2 \sum_{j=1}^{\varkappa}\left(\cdot, h_{j}\right) h_{j}-I=P-Q
$$

where $P=\sum_{j=1}^{\varkappa}\left(\cdot, h_{j}\right) h_{j}$ is the orthogonal projector $\mathbb{C}^{M-L}$ onto $\operatorname{ker}\left(\mathcal{S}_{(22)}(\tau)-1\right)$ and $Q=$ $I-P$. Moreover, for $\mu \rightarrow \tau+0$, it follows from (4.46) that

$$
S_{(22)}(\mu)-P+Q=O\left(|\mu-\tau|^{1 / 2}\right) .
$$

In accordance with (4.29),

$$
S_{(21)}(\mu)=\left(\mathcal{S}_{22}(\mu)+I_{M-L} d^{+} / d^{-}\right)^{-1} \mathcal{S}_{(21)} / d^{-} .
$$


Taking account of (4.42) and of $d^{-}=(\lambda-1) / 2 \sqrt{\lambda}$, we obtain

$$
S_{(21)}(\mu)=\left(\Gamma(\lambda) \mathcal{S}_{(21)}(\mu)+O(|\lambda|)\right) 2 \sqrt{\lambda} /(\lambda-1) .
$$

Consequently,

$$
S_{(21)}(\mu)=O\left(|\mu-\tau|^{1 / 4}\right) \rightarrow 0 \quad \text { for } \quad \mu \rightarrow \tau+0 .
$$

It remains to find the limit of $S_{(12)}(\mu)$. By virtue of $(4.28)$,

$$
S_{(12)}(\mu)=\mathcal{S}_{(12)}(\mu) d^{+}\left(I-\left(\mathcal{S}_{(22)}(\mu)+d^{+} / d^{-}\right)^{-1}\left(\mathcal{S}_{(22)}(\mu)+d^{-} / d^{+}\right)\right) .
$$

Since

$$
\left(\mathcal{S}_{(22)}(\mu)+d^{+} / d^{-}\right)^{-1}\left(\mathcal{S}_{(22)}(\mu)+d^{-} / d^{+}\right)=I-\frac{4 \lambda}{\lambda^{2}-1}\left(\mathcal{S}_{(22)}(\mu)+d^{+} / d^{-}\right)^{-1}
$$

we arrive at

$$
S_{(12)}(\mu)=\frac{2 \sqrt{\lambda}}{\lambda-1} \mathcal{S}_{(12)}(\mu)\left(-\frac{1}{2 \lambda} \sum\left(\cdot, h_{j}\right) h_{j}+\Gamma(\lambda)\right) .
$$

Recall that $h_{j} \in \operatorname{ker}\left(\mathcal{S}_{22}(\tau)-1\right) \subset \operatorname{ker}_{12}(\tau)($ see $(3.20)), \mathcal{S}_{(12)}(\mu)=\mathcal{S}_{(12)}(\tau)+O(|\mu-\tau|)$, and $\mu-\tau=\lambda^{2}$. Therefore, as $\mu \rightarrow \tau+0$ we have

$$
S_{(12)}(\mu)=O\left(|\mu-\tau|^{1 / 4}\right) \rightarrow 0
$$

\section{Method for computing the scattering matrix}

We first recall the method for the scattering matrix $S(\mu)$ in Theorem 3.6, $i$ ) with $\mu^{\prime} \leqslant \mu \leqslant \mu^{\prime \prime}$, where $\left[\mu^{\prime}, \mu^{\prime \prime}\right] \subset\left(\tau^{\prime}, \tau\right)$ or $\left[\mu^{\prime}, \mu^{\prime \prime}\right] \subset\left(\tau, \tau^{\prime \prime}\right)$. The interval $\left[\mu^{\prime}, \mu^{\prime \prime}\right]$ may contain eigenvalues of the operator (3.5). The method was justified for the Laplace operator in [2] and generalized for elliptic systems in [3]. We set

$$
\begin{aligned}
& \Pi_{+}^{r, R}=\left\{\left(y^{r}, t^{r}\right) \in \Pi^{r}: t^{r}>R\right\}, \quad G^{R}=G \backslash \cup_{r=1}^{\mathcal{T}} \Pi_{+}^{r, R}, \\
& \partial G^{R} \backslash \partial G=\Gamma^{R}=\cup_{r} \Gamma^{r, R}, \quad \Gamma^{r, R}=\left\{\left(y^{r}, t^{r}\right) \in \Pi^{r}: t^{r}=R\right\}
\end{aligned}
$$

for large $R$ and introduce the boundary value problem

$$
\begin{aligned}
-\Delta X_{j}^{R}(x, \mu)-\mu X_{j}^{R}(x, \mu) & =0, \quad x \in G^{R} \\
X_{j}^{R}(x, \mu) & =0 \quad x \in \partial G^{R} \backslash \Gamma^{R} \\
\left(-\partial_{n}+i \zeta\right) X_{j}^{R}(x, \mu) & =\left(-\partial_{n}+i \zeta\right)\left(u_{j}^{+}(x, \mu)+\sum_{k=1}^{M} a_{k} u_{k}^{-}(x, \mu)\right), x \in \Gamma^{R}(5.1
\end{aligned}
$$

where $\zeta \in \mathbb{R} \backslash\{0\}$ is an arbitrary fixed number, $a_{k}$ are complex numbers, and $u_{j}^{ \pm}$are the waves in (2.6). As an approximation to the row $\left(S_{j 1}, \ldots, S_{j M}\right)$ there serves a minimizer $a^{0}(R, \mu)=\left(a_{1}^{0}(R, \mu), \ldots, a_{M}^{0}(R, \mu)\right)$ of the functional

$$
J_{j}^{R}\left(a_{1}, \ldots, a_{M} ; \mu\right)=\left\|X_{j}^{R}(\cdot, \mu)-u_{j}^{+}(\cdot, \mu)-\sum_{k=1}^{M} a_{k} u_{k}^{-}(\cdot, \mu) ; L_{2}\left(\Gamma^{R}\right)\right\|^{2},
$$


where $X_{j}^{R}$ is a solution to problem (5.1). To clarify the dependence of $X_{j}^{R}$ on the parameters $a_{1}, \ldots, a_{M}$, we consider the problems

$$
\begin{aligned}
-\Delta v_{j}^{ \pm}-\mu v_{j}^{ \pm} & =0, \quad x \in G^{R}, \\
v_{j}^{ \pm} & =0, \quad x \in \partial G^{R} \backslash \Gamma^{R} ; \\
\left(-\partial_{n}+i \zeta\right) v_{j}^{ \pm} & =\left(-\partial_{n}+i \zeta\right) u_{j}^{ \pm}, x \in \Gamma^{R}, \quad j=1, \ldots, M ;
\end{aligned}
$$

we have $X_{j}^{R}=v_{j, R}^{+}+\sum_{k} a_{k} v_{k, R}^{-}$. Let us introduce the $M \times M$-matrices with entries

$$
\begin{aligned}
& \mathbf{E}_{j k}^{R}=\left(v_{j}^{-}-u_{j}^{-}, v_{k}^{-}-u_{k}^{-}\right)_{\Gamma^{R}}, \\
& \mathbf{F}_{j k}^{R}=\left(v_{j}^{+}-u_{j}^{+}, v_{k}^{-}-u_{k}^{-}\right)_{\Gamma^{R}} .
\end{aligned}
$$

We also set

$$
\mathbf{G}_{j}^{R}=\left(v_{j}^{+}-u_{j}^{+}, v_{j}^{+}-u_{j}^{+}\right)_{\Gamma^{R}} .
$$

Now functional (5.2) can be written in the form

$$
J_{j}^{R}(a)=\left\langle a \mathbf{E}^{R}, a\right\rangle+2 \operatorname{Re}\left\langle\mathbf{F}_{j}^{R}, a\right\rangle+\mathbf{G}_{j}^{R},
$$

where $\mathbf{F}_{j}^{R}$ is the $j$-th row of the matrix $\mathbf{F}^{R}$ and $\langle\cdot, \cdot\rangle$ is the inner product on $\mathbb{C}^{M}$. The minimizer $a^{0}(R, \mu)$ satisfies $a^{0}(R, \mu) \mathbf{E}^{R}+\mathbf{F}_{j}^{R}=0$; the matrix $\mathbf{E}^{R}$ is non-singular.

It was shown in [2] that the minimizer $a^{0}(R, \mu)$ tends to the row $\left(S_{j 1}, \ldots, S_{j M}\right)$ as $R \rightarrow+\infty$ at exponential rate. More precisely, the estimate

$$
\sum_{k=1}^{M}\left|S_{j k}(\mu)-a_{k}^{0}(R, \mu)\right| \leqslant C e^{-\delta R}
$$

holds for any $R \geqslant R_{0}$, where $\delta$ is the number in (3.3), $R_{0}$ is a sufficiently large positive number, and the constant $C$ is independent of $R$ and $\mu \in\left[\mu^{\prime}, \mu^{\prime \prime}\right]$.

We now proceed to calculating the matrix $\mathcal{S}(\mu)$ in Theorem $3.6, i i)$ with $\mu \in\left[\mu^{\prime}, \mu^{\prime \prime}\right] \subset$ $\left(\tau^{\prime}, \tau^{\prime \prime}\right)$. The interval $\left[\mu^{\prime}, \mu^{\prime \prime}\right]$ may contain the threshold $\tau$ as well as some eigenvalues of the operator (3.10). Introduce the boundary value problem

$$
\begin{aligned}
-\Delta \mathcal{X}_{j}^{R}-\mu \mathcal{X}_{j}^{R} & =0, \quad x \in G^{R} \\
\mathcal{X}_{j}^{R} & =0 \quad x \in \partial G^{R} \backslash \Gamma^{R} \\
\left(-\partial_{n}+i \zeta\right) \mathcal{X}_{j}^{R} & =\left(-\partial_{n}+i \zeta\right)\left(w_{j}^{+}+\sum_{k=1}^{M} a_{k} w_{k}^{-}\right), x \in \Gamma^{R},
\end{aligned}
$$

where $w_{j}^{ \pm}$is stable basis $(2.10),(2.11)$ in the space of waves, $\zeta \in \mathbb{R} \backslash\{0\}$, and $a_{k} \in$ $\mathbb{C}$. As an approximation to the row $\left(\mathcal{S}_{j 1}, \ldots, \mathcal{S}_{j M}\right)$, we suggest a minimizer $a^{0}(R)=$ $\left(a_{1}^{0}(R), \ldots, a_{M}^{0}(R)\right)$ of the functional

$$
\mathcal{J}_{j}^{R}\left(a_{1}, \ldots, a_{M}\right)=\left\|\mathcal{X}_{j}^{R}-w_{j}^{+}-\sum_{k=1}^{M} a_{k} w_{k}^{-} ; L_{2}\left(\Gamma^{R}\right)\right\|^{2},
$$


where $\mathcal{X}_{j}^{R}$ is a solution of problem (5.4). Let us consider the problems

$$
\begin{aligned}
-\Delta z_{j}^{ \pm}-\mu z_{j}^{ \pm} & =0, \quad x \in G^{R} ; \\
z_{j}^{ \pm} & =0, \quad x \in \partial G^{R} \backslash \Gamma^{R} ; \\
\left(-\partial_{n}+i \zeta\right) z_{j}^{ \pm} & =\left(-\partial_{n}+i \zeta\right) w_{j}^{ \pm}, x \in \Gamma^{R} ; \quad j=1, \ldots, M,
\end{aligned}
$$

set

$$
\begin{aligned}
\mathcal{E}_{j k}^{R} & =\left(z_{j}^{-}-w_{j}^{-}, z_{k}^{-}-w_{k}^{-}\right)_{\Gamma^{R}}, \\
\mathcal{F}_{j k}^{R} & =\left(z_{j}^{+}-w_{j}^{+}, z_{k}^{-}-w_{k}^{-}\right)_{\Gamma^{R}}, \\
\mathcal{G}_{j}^{R} & =\left(z_{j}^{+}-w_{j}^{+}, z_{j}^{+}-w_{j}^{+}\right)_{\Gamma^{R}},
\end{aligned}
$$

and rewrite functional (5.5) in the form

$$
\mathcal{J}_{j}^{R}(a)=\left\langle a \mathcal{E}^{R}, a\right\rangle+2 \operatorname{Re}\left\langle\mathcal{F}_{j}^{R}, a\right\rangle+\mathcal{G}_{j}^{R},
$$

where $\mathcal{F}_{j}^{R}$ is the $j$-th row of the matrix $\mathcal{F}^{R}$. Thus the minimizer $a^{0}(R)$ is a solution to the system $a^{0}(R) \mathcal{E}^{R}+\mathcal{F}_{j}^{R}=0$.

The justification of the method is similar to that in [3]. The following Propositions 5.1 and 5.2 can be verified in the same way as the analogous assertions in [3].

Proposition 5.1. The matrix $\mathcal{E}^{R}(\mu)$ with entries (5.6) is non-singular for all $\mu \in\left[\mu^{\prime}, \mu^{\prime \prime}\right]$ and $R \geqslant R_{0}$, where $R_{0}$ is sufficiently large number.

Proposition 5.2. Let $u$ be a solution to the problem

$$
\begin{aligned}
-\Delta u-\mu u & =0, \quad x \in G^{R}, \\
u & =0 \quad x \in \partial G^{R} \backslash \Gamma^{R}, \\
\left(-\partial_{n}+i \zeta\right) u & =h, x \in \Gamma^{R},
\end{aligned}
$$

with $h \in L_{2}\left(\Gamma^{R}\right)$. Then

$$
\left\|u ; L_{2}\left(\Gamma^{R}\right)\right\| \leqslant \frac{1}{|\zeta|}\left\|h ; L_{2}\left(\Gamma^{R}\right)\right\| .
$$

Proposition 5.3. Let $a^{0}(R, \mu)=\left(a_{1}^{0}(R, \mu), \ldots, a_{M}^{0}(R, \mu)\right)$ be a minimizer of the functional $\mathcal{J}_{l}^{R}$ in (5.5). Then

$$
\mathcal{J}_{l}^{R}\left(a^{0}(R, \mu)\right) \leqslant C e^{-2 \gamma R} \text { for } R \rightarrow \infty,
$$

where the constant $C$ is independent of $R \geqslant R_{0}, \mu \in\left[\mu^{\prime}, \mu^{\prime \prime}\right]$, and $\gamma=\gamma(\mu)$ is the piecewise constant index described in Lemma 3.4. For all $R \geqslant R_{0}$ and $\mu \in\left[\mu^{\prime}, \mu^{\prime \prime}\right]$ the components of the vector $a^{0}(R, \mu)$ are uniformly bounded,

$$
\left|a_{j}^{0}(R, \mu)\right| \leqslant \text { const }<\infty, \quad j=1, \ldots, M .
$$

Proof. Relation (5.8) has been obtained in the same manner as in [3]. Let us verify the uniform boundedness of the minimizer $a^{0}(R, \mu)$. According to Lemma 3.4, for $\left[\mu^{\prime}, \mu^{\prime \prime}\right]$ there exists a finite covering $I_{p}$ such that for each interval $I_{p}$ one can choose a number $\gamma(\mu)$ in 
(3.8) (and consequently in (5.8)) independent of $\mu$. Moreover, $\max _{\mu \in I_{p}} \operatorname{Re} \sqrt{\tau-\mu}<\gamma<$ $\min _{\mu \in I_{p}} \operatorname{Re} \sqrt{\tau^{\prime \prime}-\mu}$. We consider that $\mu$ runs through one of the covering intervals. Denote by $Z_{l}^{R}$ the solution of problem (5.4) corresponding to $a^{0}(R, \mu)=\left(a_{1}^{0}(R, \mu), \ldots, a_{M}^{0}(R, \mu)\right)$. Setting $u=v=Z_{l}^{R}$ in the Green formula, we obtain

$$
\left(-\partial_{\nu} Z_{l}^{R}, Z_{l}^{R}\right)_{\Gamma^{R}}-\left(Z_{l}^{R},-\partial_{\nu} Z_{l}^{R}\right)_{\Gamma^{R}}=0 .
$$

By virtue of (5.8),

$$
\left\|Z_{l}^{R}-\left(w_{l}^{+}+\sum_{j=1}^{M} a_{j}(R, \mu) w_{j}^{-}\right) ; L_{2}\left(\Gamma^{R}\right)\right\|=O\left(e^{-\gamma R}\right), \quad R \rightarrow \infty,
$$

uniformly with respect to $\mu$. Since

$$
\left.\left(-\partial_{\nu}+i \zeta\right) Z_{l}^{R}\right|_{\Gamma^{R}}=\left.\left(-\partial_{\nu}+i \zeta\right)\left(w_{l}^{+}+\sum_{j=1}^{M} a_{j}^{0}(R) w_{j}^{-}\right)\right|_{\Gamma^{R}},
$$

from (5.9) it follows that

$$
\left\|-\partial_{\nu}\left(Z_{l}^{R}-\left(w_{l}^{+}+\sum_{j=1}^{M} a_{j}^{0}(R) w_{j}^{-}\right)\right) ; L_{2}\left(\Gamma^{R}\right)\right\|=O\left(e^{-\gamma R}\right), \quad R \rightarrow \infty .
$$

Recall that for $\mu>\tau$, the waves $w_{l}^{ \pm}$are bounded functions; for $\mu<\tau$ the waves $w_{l}^{ \pm}$with $L<l \leqslant M$ defined by (4.2) grow at infinity as $O\left(e^{\sqrt{\tau-\mu}|x|}\right)$ and as $O(|x|)$ for $\mu=\tau$. We use (5.10) and (5.11) to reduce (5.9) to the form

$$
\left(-\partial_{\nu} \varphi_{l}, \varphi_{l}\right)_{\Gamma^{R}}-\left(\varphi_{l},-\partial_{\nu} \varphi_{l}\right)_{\Gamma^{R}}=\left|a^{0}(R)\right| O\left(e^{-(\gamma-\sqrt{\tau-\mu}-\varepsilon) R}\right),
$$

where $\varphi_{l}=w_{l}^{+}+\sum a_{j}^{0}(R) w_{j}^{-}$; as before, $\sqrt{\tau-\mu}=i \sqrt{\mu-\tau}$ for $\mu>\tau$, $\varepsilon$ being an arbitrary small positive number. In view of (2.12) and (2.13), the left-hand side is equal to $-i(1-$ $\left.\sum\left|a_{j}^{0}(R)\right|^{2}\right)$. Therefore,

$$
\left|a^{0}(R)\right|^{2}=1+o\left(\left|a^{0}(R)\right|\right),
$$

which leads to $\left|a^{0}(R)\right|=1+o(1)$. Looking over all elements of the covering, we obtain the desired estimate everywhere on $\left[\mu^{\prime}, \mu^{\prime \prime}\right]$.

Theorem 5.4. For all $R \geqslant R_{0}$, where $R_{0}$ is a sufficiently large number, and for all $\mu \in$ $\left[\mu^{\prime}, \mu^{\prime \prime}\right] \subset\left(\tau^{\prime}, \tau^{\prime \prime}\right)$ there exists a unique minimizer $a^{0}(R, \mu)=\left(a_{1}^{0}(R, \mu), \ldots, a_{M}^{0}(R, \mu)\right)$ of the functional $\mathcal{J}_{l}^{R}$ in (5.2). The estimates

$$
\sum_{k=1}^{M}\left|\mathcal{S}_{j k}(\mu)-a_{k}^{0}(R, \mu)\right| \leqslant C e^{-\Lambda R}
$$

hold for all $R \geqslant R_{0}, \mu \in\left[\mu^{\prime}, \mu^{\prime \prime}\right]$, and $0<\Lambda<\min _{\mu \in\left[\mu^{\prime}, \mu^{\prime \prime}\right]} \operatorname{Re}\left(\sqrt{\tau^{\prime \prime}-\mu}-\sqrt{\tau-\mu}\right)$, where $\sqrt{\tau-\mu}=i \sqrt{\mu-\tau}$ for $\mu>\tau$ and the constant $C=C(\Lambda)$ is independent of $R$ and $\mu$. 
Proof. As in the proof of the previous assertion, we assume that $\mu$ runs through an interval $I_{p}$ of the covering of $\left[\mu^{\prime}, \mu^{\prime \prime}\right]$ in Lemma 3.4, so the number $\gamma$ in (3.8), (5.10) and (5.11) is independent of $\mu$, while $\max _{\mu \in I_{p}} \operatorname{Re} \sqrt{\tau-\mu}<\gamma<\min _{\mu \in I_{p}} \operatorname{Re} \sqrt{\tau^{\prime \prime}-\mu}$.

Let $Y_{l}^{R}$ be a solution to problem (5.4), where $a_{j}, j=1, \ldots, M$, are taken to be the entries $\mathcal{S}_{l j}$ of the scattering matrix $\mathcal{S}$, and let $Z_{l}^{R}$ and $\left(a_{1}^{0}(R, \mu), \ldots, a_{M}^{0}(R, \mu)\right)$ be the same as in Proposition 5.3. We substitute $u=v=U_{l}:=Y_{l}-Z_{l}^{R}$ into the Green formula. Since $U_{l}$ satisfies the first two equations in (5.4), we have

$$
\left(-\partial_{\nu} U_{l}, U_{l}\right)_{\Gamma^{R}}-\left(U_{l},-\partial_{\nu} U_{l}\right)_{\Gamma^{R}}=0 .
$$

Setting

$$
\varphi_{l}=w_{l}^{+}+\sum_{j=1}^{M} a_{j}^{0}(R, \mu) w_{j}^{-}, \quad \psi_{l}=w_{l}^{+}+\sum_{j=1}^{M} S_{l j}(\mu) w_{j}^{-},
$$

we write down $U_{l}$ in the form

$$
U_{l}=Y_{l}-Z_{l}^{R}=\left(Y_{l}-\psi_{l}\right)+\left(\psi_{l}-\varphi_{l}\right)+\left(\varphi_{l}-Z_{l}^{R}\right) .
$$

Note that $\left.\left(Y_{l}-\psi_{l}\right)\right|_{\Gamma^{R}}=O\left(e^{-\gamma R}\right)$ by virtue (3.8). Moreover, by Proposition 5.3, the components of the minimizer $a_{j}(R, \mu)$ are uniformly bounded. In view of (5.10)and (5.11), this leads from (5.13) to the relation

$$
\left(-\partial_{\nu}\left(\psi_{l}-\varphi_{l}\right),\left(\psi_{l}-\varphi_{l}\right)\right)_{\Gamma^{R}}-\left(\left(\psi_{l}-\varphi_{l}\right),-\partial_{\nu}\left(\psi_{l}-\varphi_{l}\right)\right)_{\Gamma^{R}}=O\left(e^{-(\gamma-\sqrt{\tau-\mu}-\varepsilon) R}\right),
$$

where $\varepsilon$ is an arbitrary small positive number. Straightforward calculation shows that the left-hand side is equal to $i \sum_{j=1}^{M}\left|a_{j}^{0}(R, \mu)-\mathcal{S}_{l j}(\mu)\right|^{2}$ (it suffices to use (5.13), (2.12), and (2.13)). Hence

$$
\sum_{j=1}^{M}\left|a_{j}^{0}(R, \mu)-\mathcal{S}_{l j}(\mu)\right|^{2}=O\left(e^{-(\gamma-\sqrt{\tau-\mu}-\varepsilon) R}\right)
$$

and we arrive at (5.12) for $\mu \in I_{p}$ и $\Lambda \leqslant \min _{\mu \in I_{p}}(\gamma-\operatorname{Re} \sqrt{\tau-\mu}-\varepsilon) / 2$.

We now prove that the inequality

$$
\sum_{j=1}^{M}\left|a_{j}(R, \mu)-\mathcal{S}_{l j}(\mu)\right|^{2}=O\left(e^{-2(\gamma-\sqrt{\tau-\mu}-\varepsilon)\left(1-2^{-N}\right) R}\right)
$$

holds for any positive integer $N$. For $N=1$ the inequality has been obtained, so it suffices to derive from (5.16) the same estimate with $N+1$ instead of $N$. We have

$$
\psi_{l}-\varphi_{l}=\sum_{j=1}^{M}\left(\mathcal{S}_{l j}(\mu)-a_{j}(R, \mu)\right) w_{j}^{-}=O\left(e^{-\left[(\gamma-\sqrt{\tau-\mu})\left(1-2^{-N}\right)-\sqrt{\tau-\mu}-\varepsilon\right] R}\right) .
$$

So we can go from (5.13) to (5.15) with right-hand side replaced by $O\left(e^{z}\right)$ with $z=-[(\gamma-$ $\left.\sqrt{\tau-\mu}-\varepsilon)\left(1-2^{-N}\right)-\sqrt{\tau-\mu}-\varepsilon+\gamma\right] R=-2(\gamma-\sqrt{\tau-\mu}-\varepsilon)\left(1-2^{-N-1}\right) R$. Again calculating the left hand-side of (5.15), we obtain

$$
\sum_{j=1}^{M}\left|a_{j}(R, \mu)-\mathcal{S}_{l j}(\mu)\right|^{2}=O\left(e^{-2(\gamma-\sqrt{\tau-\mu}-\varepsilon)\left(1-2^{-N-1}\right) R}\right)
$$


Hence we have proved (5.12) for any positive integer $N$ with $\mu \in I_{p}$ and $\Lambda \leqslant \min _{\mu \in I_{p}}(\gamma-$ $\operatorname{Re} \sqrt{\tau-\mu}-\varepsilon)\left(1-2^{-N}\right)$. Increasing $N$ and decreasing $\varepsilon$, we can take $\Lambda$ to be arbitrarily close to $\gamma-\operatorname{Re} \sqrt{\tau-\mu}$. Looking over all intervals $I_{p}$ of the covering, we obtain the needed estimate everywhere on $\left[\mu^{\prime}, \mu^{\prime \prime}\right]$ with $\Lambda<\min _{\mu \in\left[\mu^{\prime}, \mu^{\prime \prime}\right]}(\gamma(\mu)-\operatorname{Re} \sqrt{\tau-\mu})$. Finally, for the difference between $\gamma(\mu)$ and $\operatorname{Re} \sqrt{\tau^{\prime \prime}-\mu}$ to be so small as needed, it suffices to refine the covering of $\left[\mu^{\prime}, \mu^{\prime \prime}\right]$.

In a neighborhood of the threshold $\tau$, the matrix $\mathcal{S}(\mu)$ can be calculated by the method presented in this paper. Since the limits of $S(\mu)$ as $\mu \rightarrow \tau \pm 0$ are finite, the connection between $S(\mu)$ and $\mathcal{S}(\mu)$ allows to calculate $S(\mu)$ for $\mu$ in vicinity of $\tau$.

\section{References}

[1] Nazarov S.A. and Plamenevsky B. A., Elliptic Problems in Domains with Piecewise Smooth Boundaries, Berlin; New-York: De Gruyter, 1994.

[2] Plamenevskii B. A. and Sarafanov O.V., On a method for computing waveguide scattering matrices, St.Petersburg Math.J., 23 (2012), no 1, 139 - 160.

[3] Plamenevskii B. A. and Sarafanov O.V., On a method for computing waveguide scattering matrices in the presence of point spectrum, Functional Analysis and Its Applications, 48 (2014), no 1, $49-58$.

[4] M. Costabel and M. Dauge, Stable asymptotics for elliptic systems on plane domains with corners, Comm. Partial Differential Equations, 19 (1994), no 9-10, 1677-1726.

[5] Maz'ya, V. and Rossmann, J. On a problem of Babuška (stable asymptotics of the solution to the Dirichlet problem for elliptic equations of second order in domains with angular points), Math. Nachr. 155 (1993), 199-220.

[6] Kamotskii I. V. and Nazarov S.A., Wood's anomalies and surface waves in the problem of scattering by a periodic boundary, Sbornik: Mathematics, 190 (1999), no 1, 111-141; 190 (1999), no 2, 205- 231.

[7] Nazarov S.A. and Plamenevskii B. A., Self-adjoint problems with radiation conditions at edges of the boundary, St.Petersburg Math.J., 4 (1992), no 3, 569 - 594.

[8] Kamotskii I. V. and Nazarov S.A., Augmented scattering matrix and exponentially decaying solutions of an elliptic problem in a cylindrical domain, J. Math. Sciences, 111 (2002),no 4, $3657-3666$.

[9] Gohberg I., Goldberg S., and Kaashoek M.A., Classes of Linear Operators, v.1, Oper. Theory: Adv. Appl. 49, Birkhäuser, Basel-Boston-Berlin.

[10] Gohberg I. Ts. and Sigal E.I., An operator generalization of the logarithmic residue theorem and the theorem of Rouché, Math.USSR-Sbornik, 13, (1971), c. 603-625. 